\title{
B.1.1.529 escapes the majority of SARS-CoV-2 neutralizing antibodies of diverse epitopes
}

Xiaoliang Xie ( $\nabla$ sunneyxie@biopic.pku.edu.cn )

Peking University https://orcid.org/0000-0001-9281-5239

\section{Yunlong Cao}

Peking University https://orcid.org/0000-0001-5918-1078

\section{jing wang}

https://orcid.org/0000-0002-9084-9985

\section{Fanchong Jian}

Biomedical Pioneering Innovation Center (BIOPIC), Peking University, Beijing, P.R. China. https://orcid.org/0000-0001-8703-3507

\section{Tianhe Xiao}

Biomedical Pioneering Innovation Center (BIOPIC), Peking University, Beijing, P.R. China.

\section{Weiliang Song}

Biomedical Pioneering Innovation Center (BIOPIC), Peking University, Beijing, P.R. China.

\section{Ayijiang Yisimayi}

Peking University

Tao Li

National Institutes for Food and Drug Control

\section{Yuanling Yu}

National Institutes for Food and Drug Control

\section{Yao Wang}

Biomedical Pioneering Innovation Center (BIOPIC), Peking University, Beijing, P.R. China.

\section{Jing Wang}

Biomedical Pioneering Innovation Center (BIOPIC), Peking University, Beijing, P.R. China.

\section{Xiao Niu}

Biomedical Pioneering Innovation Center (BIOPIC), Peking University, Beijing, P.R. China.

\section{Peng Wang}

Biomedical Pioneering Innovation Center (BIOPIC), Peking University, Beijing, P.R. China.

\section{Ran An}

Biomedical Pioneering Innovation Center (BIOPIC), Peking University, Beijing, P.R. China. Hui Liang

Biomedical Pioneering Innovation Center (BIOPIC), Peking University, Beijing, P.R. China.

\section{Haiyan Sun}

Biomedical Pioneering Innovation Center (BIOPIC), Peking University, Beijing, P.R. China. 


\section{Sijie Yang}

Biomedical Pioneering Innovation Center (BIOPIC), Peking University, Beijing, P.R. China.

\section{Qianqian Cui}

National Institutes for Food and Drug Control

\section{Shuo Liu}

National Institute for Food and Drug Control

\section{Shuo Du}

Peking University https://orcid.org/0000-0003-0936-0785

\section{Zhiying Zhang}

Peking University https://orcid.org/0000-0002-5690-9796

\section{Fei Shao}

Biomedical Pioneering Innovation Center (BIOPIC), Peking University, Beijing, P.R. China.

\section{Weijin Huang}

National Institutes for Food and Drug Control (NIFDC) https://orcid.org/0000-0002-4246-8889

\section{Junyu Xiao}

Peking University https://orcid.org/0000-0003-1822-1701

\section{Youchun Wang}

National Institutes for Food and Drug Control https://orcid.org/0000-0001-9769-5141

\section{Xiangxi Wang}

Institute of Biophysics https://orcid.org/0000-0003-0635-278X

\section{Xiaodong Yang}

Beijing Youan Hospital, Beijing, P.R. China.

\section{Qianqian Li}

National Institutes for Food and Drug Control

\section{Xiaohua Hao}

Beijing Ditan Hospital

Jin Ronghua

Beijing Ditan hospital

\section{Biological Sciences - Article}

\section{Keywords:}

Posted Date: December 8th, 2021

DOI: https://doi.org/10.21203/rs.3.rs-1148985/v1

License: (c) (i) This work is licensed under a Creative Commons Attribution 4.0 International License. Read Full License 
Version of Record: A version of this preprint was published at Nature on December 23rd, 2021. See the published version at https://doi.org/10.1038/s41586-021-04385-3. 
1 B.1.1.529 escapes the majority of SARS-CoV-2 neutralizing antibodies of

\section{diverse epitopes}

Yunlong Cao ${ }^{1,2, \#, *}$, Jing Wang ${ }^{1,3, \#,}$, Fanchong Jian ${ }^{1,4, \#}$, Tianhe Xiao ${ }^{1,5, \#}$, Weiliang

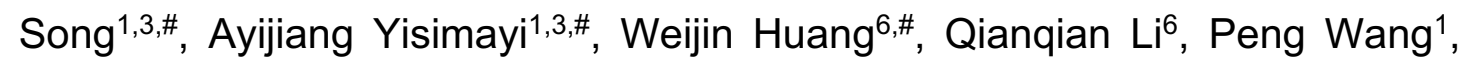
Ran $\mathrm{An}^{1}$, Jing Wang ${ }^{1}$, Yao Wang ${ }^{1}$, Xiao Niu ${ }^{1,4}$, Sijie Yang ${ }^{1,7}$, Hui Liang ${ }^{1}$, Haiyan Sun ${ }^{1}$, Tao Li ${ }^{6}$, Yuanling Yu', Qianqian Cui ${ }^{6}$, Shuo $\mathrm{Liu}^{6}$, Xiaodong Yang ${ }^{8}$, Shuo $\mathrm{Du}^{3}$, Zhiying Zhang ${ }^{3}$, Xiaohua $\mathrm{Hao}^{9}$, Fei Shao ${ }^{1}$, Ronghua $\mathrm{Jin}^{9}$, Xiangxi Wang ${ }^{10,{ }^{*}}$, Junyu Xiao ${ }^{2,3,{ }^{*}}$, Youchun Wang ${ }^{6,{ }^{*}}$, Xiaoliang Sunney $\mathrm{Xie}^{1,2,{ }^{*}}$

${ }^{1}$ Biomedical Pioneering Innovation Center (BIOPIC), Peking University, Beijing, P.R. China.

${ }^{2}$ Beijing Advanced Innovation Center for Genomics (ICG), Peking University, Beijing, P.R. China.

${ }^{3}$ School of Life Sciences, Peking University, Beijing, P.R. China.

${ }^{4}$ College of Chemistry and Molecular Engineering, Peking University, Beijing, P.R. China.

${ }^{5}$ Joint Graduate Program of Peking-Tsinghua-NIBS, Academy for Advanced Interdisciplinary Studies, Peking University, Beijing, China.

${ }^{6}$ Division of HIVIAIDS and Sex-transmitted Virus Vaccines, Institute for Biological Product Control, National Institutes for Food and Drug Control (NIFDC), and WHO Collaborating Center for Standardization and Evaluation of Biologicals, Beijing, P.R. China.

${ }^{7}$ Tsinghua-Peking Center for Life Sciences, Beijing, P.R. China.

${ }^{8}$ Beijing YouAn Hospital, Capital Medical University, Beijing, P.R. China.

${ }^{9}$ Beijing Ditan Hospital, Capital Medical University, Beijing, P.R. China.

${ }^{10}$ CAS Key Laboratory of Infection and Immunity, National Laboratory of Macromolecules, Institute of Biophysics, Chinese Academy of Sciences, Beijing, P.R. China.

"Correspondence: Yunlong Cao (yunlongcao@pku.edu.cn); Junyu Xiao (junyuxiao@pku.edu.cn); Xiangxi Wang (xiangxi@ibp.ac.cn); Youchun Wang (wangyc@nifdc.org.cn); Xiaoliang Sunney Xie (sunneyxie@biopic.pku.edu.cn) \#These authors contributed equally. 


\section{Abstract}

The SARS-CoV-2 B.1.1.529 variant (Omicron) contains 15 mutations on the receptor-binding domain (RBD). How Omicron would evade RBD neutralizing antibodies (NAbs) and humoral immunity requires immediate investigation. Here, we used high-throughput yeast display screening ${ }^{1,2}$ to determine the RBD escaping mutation profiles for 247 human anti-RBD NAbs identified from SARSCoVISARS-CoV-2 convalescents and vaccinees. Based on the results, NAbs could be unsupervised clustered into six epitope groups (A-F), which is highly concordant with knowledge-based structural classifications ${ }^{3-5}$. Strikingly, various single mutations of Omicron could impair NAbs of different epitope groups. Specifically, NAbs in Group A-D, whose epitope overlaps with ACE2binding motif, are largely escaped by K417N, N440K, G446S, E484A, Q493K, and G496S. Group E (S309 site) ${ }^{6}$ and F (CR3022 site) ${ }^{7}$ NAbs, which often exhibit broad sarbecovirus neutralizing activity, are less affected by Omicron, but still, a subset of NAbs are escaped by G339D, S371L, and S375F. Furthermore, B.1.1.529 pseudovirus neutralization and RBD binding assay showed that single mutation tolerating NAbs could also be escaped due to multiple synergetic mutations on their epitopes. In total, over $85 \%$ of the tested NAbs are escaped by Omicron. Regarding NAb drugs, LY-CoV016/LY-CoV555 cocktail, REGN-CoV2 cocktail, AZD1061/AZD8895 cocktail, and BRII-196 were escaped by Omicron, while VIR7831 and DXP-604 still function at reduced efficacy. Together, data suggest Omicron could cause significant humoral immune evasion, while NAbs targeting the sarbecovirus conserved region remain most effective. Our results offer instructions for developing NAb drugs and vaccines against Omicron and future variants. 
The SARS-CoV-2 B.1.1.529 variant was first reported to the World Health Organization (WHO) on 24 November 2021. It appears to be rapidly spreading, and the WHO classified it as a variant of concern (VOC) only two days after, designating it as Omicron ${ }^{8,9}$. An unusually large number of mutations are found in Omicron, including over 30 in the spike protein ${ }^{10}$ (Fig. 1a). The receptorbinding domain, responsible for interacting with the ACE2 receptor, bears 15 of these mutations, including G339D, S371L, S373P, S375F, K417N, N440K, G446S, S477N, T478K, E484A, Q493R, G496S, Q498R, N501Y, and Y505H. Some of these mutations are very concerning due to their well-understood functional consequences, such as $\mathrm{K} 417 \mathrm{~N}$ and N501Y, which contribute to immune escape and higher infectivity ${ }^{11-14}$. Many other mutations' functional impacts remain to be investigated.

The S protein is the target of essentially all NAbs found in the convalescent sera or elicited by vaccines. Most of the N-terminal domain (NTD) neutralizing antibodies target an antigenic "supersite" in NTD, involving the N3 (residues 141 to 156 ) and N5 (residues 246 to 260 ) loops ${ }^{15,16}$, and are thus very prone to NTD mutations. Omicron carries the $\Delta 143-145$ mutation, which would alter the N3 loop and most likely result in immune escape of most anti-NTD NAbs (Extended Data Fig. 1). Compared to NTD targeting NAbs, RBD targeting NAbs are particularly abundant and potent, and display diverse epitopes. Evaluating how Omicron affects the neutralization capability of anti-RBD NAbs of diverse classes and epitopes is urgently needed.

\section{B.1.1.529 escapes NAbs of diverse epitopes}

RBD-directed SARS-CoV-2 NAbs can be assigned into different classes or binding sites based on structural analyses by cryo-EM or high-resolution crystallography ${ }^{3-5,17}$; however, structural data only indicates the contacting amino acids, but does not infer the escaping mutations for a specific antibody. Recent advances in deep antigen mutation screening using FACS (fluorescence-activated cell sorting)-based yeast display platform has allowed 
the quick mapping of all single amino acid mutations in the RBD that affect the binding of SARS-CoV-2 RBD NAbs ${ }^{1,18}$. The method has proven highly effective in predicting $N A B$ drug efficacy toward mutations ${ }^{2}$. However, to study how human humoral immunity may react to highly mutated variants like B.1.1.529 requires mutation profiling of a large collection of NAbs targeting different regions of RBD, and FACS-based yeast display mutation screening is limited by low experimental throughput. Here we further developed a MACS (magneticactivated cell sorting) -based screening method which increases the throughput near 100-fold and could obtain comparable data quality like FACS (Fig 1b; Extended Data Fig 2). Using this method, we quickly characterized the RBD escaping mutation profile for a total of 247 NAbs (Supplymentary Files 1-6). Half of the NAbs were part of the antibodies identified by us using single-cell VDJ sequencing of antigen-specific memory B cells from SARS-CoV-2 convalescents, SARS-CoV-2 vaccinees, and SARS-CoV convalescents who recently received SARS-CoV-2 vaccines. The other half of NAbs were identified by groups worldwide and usually have antibody-antigen structures $3,5,6,12,17,19-42$ (Supplementary Files 7).

The high-throughput screening capability allowed us to classify these NAbs into six Epitope Groups (A-F) using unsupervised clustering without dependence on structural studies, and the grouping is highly concordant with the knowledgebased structural classifications ${ }^{3-5,17}$ (Fig. 1C and 1e). In particular, Group A-D NAbs largely correspond to the RBS A-D NAbs described by Yuan et al. ${ }^{4}$, and their epitopes include RBD residues involved in binding to ACE2. Group A and B NAbs, represented by CB6/LY-CoV016 and AZD8895 respectively, usually can only bind to the 'up' RBD, whereas most of the Group $C$ and $D$ members, such as LY-CoV555 and REGN10987, bind to RBDs regardless of their 'up' and 'down' conformations. Group E and F NAbs target the S309/VIR-7831 site and CR3022 site, respectively, and neutralize SARS-CoV-2 using other mechanisms than directly interfering with ACE2 binding.

Inferred from the escaping mutation profiles, various single mutations of Omicron could impair NAbs of different epitope groups (Fig 1f). Specifically, NAbs in Group A-D, whose epitope overlaps with ACE2-binding motif, are 
largely escaped by single mutations of K417N, G446S, E484A, Q493K, and G496S. Also, a subset of NAbs of Group $E$ and are escaped by single mutations of G339D, N440K, S371L, S375F. However, due to the extensive mutations accumulated on Omicron's RBD, studying NAb's response to Omicron only in the single mutation context is insufficient. Indeed, B.1.1.529 pseudovirus neutralization and Spike ELISA binding assay showed that single mutation tolerating NAbs could also be escaped by Omicron due to multiple synergetic mutations on their epitopes (Fig 1d and 1f). In total, over $85 \%$ of the tested human NAbs are escaped, suggesting that Omicron could cause significant humoral immune evasion.

\section{ACE2-blocking NAbs are more vulnerable to B.1.1.529}

It is crucial to analyze how each group of NAbs react to Omicron to instruct the development of NAb drugs and vaccines. Group A NAbs mainly contains the VH3-53NH3-66 germline gene-encoded antibodies, which are abundantly present in our current collection of SARS-CoV-2 neutralizing antibodies $19,23,24,28,43-45$, including several antibodies that have obtained emergency use authorization (CB6/LY-CoV016) ${ }^{21}$ or are currently being studied in clinical trials (P2C-1F11/BRII-196, BD-604/DXP-604) 20,46 (Fig. 2a, e). Group A NAbs often exhibit less somatic mutations and shorter CDR3 length compared to other groups (Extended Data 5a, b). The epitopes of these antibodies extensively overlap with the binding site of ACE2 and are often evaded by RBD mutations on K417, D420, F456, A475, L455 sites (Fig 2i, Extended Data Fig 3a). Most NAbs in Group A were already escaped by B.1.351 (Beta) strain, specifically K417N (Extended Data Fig 7a), due to a critical salt bridge interaction between Lys417 and a negatively charged residue in the antibody (Fig. 2i, m). The NAbs that survived Beta strain, such as BRII-196 and DXP-604, are insensitive to the $\mathrm{K} 417 \mathrm{~N}$ single site change but could also be heavily affected by the combination of K417N and other RBD mutations located on their epitopes, like S477N, Q493R, G496S, Q498R, N501Y, and Y505H of Omicron, causing lost or reduction of neutralization (Fig 2i; Extended Data Fig 4a).

The VH1-58 gene-encoded NAbs are enriched in Group B (Fig. 2b). These 
NAbs such as AZD8895 ${ }^{38}$, REGN10933 ${ }^{44}$, and BD-836 ${ }^{47}$ bind to the left shoulder of RBD, often focusing on the far tip (Fig $2 n$ ). These NAbs are very sensitive to the change of F486, N487, and G476 (Fig 2j, Extended Data Fig 3b). Fortunately, F486 and a few other major targeting sites of these NAbs are critically involved in ACE2-binding, and therefore they are generally harder to be escaped. NAbs that focus on the far tip of the left RBD shoulder, such as AZD8895 and BD-836, could survive Beta (fig 2f); however, Omicron could significantly reduce Group $B$ NAbs' binding affinity to RBD, potentially through S477N/T478K/E484A on their epitope (Extended Data Fig 4b) ${ }^{48}$, resulting in the loss of neutralization. Group B NAbs are mostly escaped by Omicron and should not be chosen for drug development against Omicron.

Group C NAbs are frequently encoded by VH1-2 and VH1-69 (Fig. 2c). The majority of NAbs in this group could bind to both "up" and "down" RBDs, resulting in higher neutralization potency compared to other groups (Extended Data Fig 5c). Several highly potent antibodies are found in Group C, including BD-368-2/DXP-593 ${ }^{46}, \mathrm{CO02}^{3}$, and LY-CoV555 ${ }^{49}$. They bind to the right shoulder of RBD (Fig 2o), and are mostly prone to the change of Glu484 (Extended Data Fig 3c, 4c), such as the E484K mutation found in Beta (Fig 2g). The E484A mutation seen in Omicron elicited a similar escaping effect, although the change to Ala is slightly subtler, and could be tolerated by certain antibodies in this group (Extended Data Fig 7b). All Group C NAbs tested are escaped by Omicron (Fig 2k).

Group D NAbs consist of diverse IGHV gene-encoded antibodies (Fig. 2d). Prominent members in this group include REGN10987 ${ }^{44}$ and AZD1061 ${ }^{38}$ (Fig 2h). They further rotate down from the RBD right shoulder towards the $\$ 309$ site when compared to Group C (Fig 2p). As a loop formed by residues 440449 in RBD is critical for the targeting of this group of NAbs, they are sensitive to the changes of Asn440, Lys444, Gly446, and Asn448 (Extended Data Fig 3d, 4d). Most NAbs of Group D remain active against Beta; however, G446S would substantially affect their neutralization capability against Omicron (Fig 2I). Also, for those NAbs that could tolerate G446S single mutation, the N440K/G446S combination may significantly reduce their binding affinity, 
resulting in that most Group D NAbs are escaped by Omicron.

\section{SARS cross-reactive NAbs are less affected by B.1.1.529}

Group E and F NAbs are rarer when compared to the other four groups. The archetypical member of each group was originally isolated from a SARS-CoV convalescent, and displays SARS-CoV-2 cross-neutralizing activity. There is no clear VDJ convergent effect compared to Group A, B, and C (Fig 3a, b), and the mutation rate and CDR3 length are larger than other groups. NAbs in Group $E$ and $F$ rarely compete with ACE2; thus, their average IC50 is higher than NAbs in Group A-D (Extended Data 5c, d, e). High-resolution structures of Group E and F NAbs are limited; thus, their epitope distribution and neutralization mechanism are not well-understood.

NAbs in Group E, such as VIR7831/S309, may recognize a mixed protein/carbohydrate epitope, involving the N-linked glycan on Asn343 6 . Inferred from the escaping mutation profiles (Fig 3c), Group E NAbs are often sensitive to changes of R346, T345, and G339 (Extended Data Fig 3e, 4e). The G339D mutation would affect a subset of NAbs' neutralization performance. Also, part of Group E NAbs' epitope would extend to the 440-449 loop, making

Group F NAbs such as CR3022 and S304 target a cryptic site in RBD that is generally not exposed (Fig $3 \mathrm{~h}, \mathrm{i}$ ), therefore their neutralizing activities are generally weaker ${ }^{7}$. Group F NAbs are often sensitive to changes of K378, T376, and F374 (Extended Data Fig 3f, 4f). A loop involving RBD residues 371375 lies in the ridge between the $E$ and F sites; therefore, a subset of Group F NAbs, including some Group E NAbs, could be affected by the S371L/S373P/S375F mutations if their epitopes extend to this region (Fig 3d, f). Interestingly, a part of Group F NAbs is highly sensitive to G504 and V503, similar to the epitopes of S2. (Fig 3j), suggesting that they can compete with ACE2. Indeed, several NAbs, such as BD55-5300 and BD55-3372, exhibited high neutralization potency compared to other NAbs in Group F. However, These antibodies' neutralization capability might be undermined by N501Y and 
$\mathrm{Y} 505 \mathrm{H}$ of Omicron.

\section{B.1.1.529 escapes the majority of NAb drugs}

As for NAb drugs, consistent with their escaping mutation profiles, LYCoV016/LY-CoV555 cocktail, REGN-10933/REGN-109876 cocktail, and AZD1061 are escaped by Omicron (Fig 4a). The binding affinity of AZD8895 and BRII-196 toward Omicron RBD is significantly reduced, potently due to multiple mutations accumulating on their epitopes, such that AZD8895 and BRII-196 failed to neutralize Omicron. VIR7831 retains strong RBD binding capability, although Gly339 is part of its epitope, the G339D mutation in Omicron does not appear to affect VIR7831's binding; however, VIR7831's IC50 is slightly reduced to $181 \mathrm{ng} / \mathrm{mL}$. DXP-604's binding affinity against Omicron RBD is largely reduced compared to wildtype RBD; nevertheless, it can still neutralize Omincron at an IC50 of $280 \mathrm{ng} / \mathrm{mL}$, a nearly 30 -fold reduction compared to wildtype (Fig 4b). Additionally, several NAbs in Group E and F have shown high potency against Omicron and broad pan-sarbecovirus neutralization ability, promising for NAb drug development (Fig 4c). Many more NAbs identified from vaccinated SARS convalescents are waiting to be characterized.

\section{Discussion}

The high-throughput yeast screening method provides a laboratory means for quickly examining the evolution outcome under a particular NAb, however, the current throughput using FACS is limited and can't be used to evaluate a large NAb library. By virtue of MACS, we are able to increase the throughput by two orders of magnitude. In doing so, we were able to gain statistical confidence for the survival proportion of anti-RBD NAbs in each epitope group against Omicron. In addition to mutations in the RBD, changes in other regions of the $S$ protein are also concerning. The $\Delta 69-70$ mutation in the $\mathrm{N}$-terminal domain (NTD) may enhance viral infectivity ${ }^{50}$. The $\mathrm{P} 681 \mathrm{H}$ mutation, located in the furin cleavage site, could promote furin cleavage and therefore accelerate cell 
To date, a large number of SARS-CoV-2 NAbs have been identified from convalescents and vaccinees. The most potent NAbs are frequently found in Groups A-D as we described above, and tend to directly interfere with the binding of ACE2. Nevertheless, the neutralizing powers of these NAbs are often abrogated by RBD mutations in the evolutionary arms race between SARSCoV-2 and human humoral immunity. Indeed, we showed that Omicron would escape from the majority of SARS-CoV-2 NAbs in this collection. Some superpotent NAbs in Group A may still function due to their extremely high affinity for RBD (such as DXP604), albeit at reduced efficacy. A subset of Group B NAbs (including AZD8895) is also more difficult to be evaded by single amino acid changes, as they target a small epitope involving several RBD residues that are essential for ACE2 binding; however, Omicron significantly reduced the binding affinity of those NAbs, possibly through multiple synergetic mutations, eventually causing lost of neutralization. On the other hand, Groups E and F NAbs are less affected by Omicron, likely because they are not abundant in population, hence exerting less evolutionary pressure for RBD to mutate in the corresponding epitope groups. These NAbs target conserved RBD regions in Sarbecovirus and therefore are ideal targets for future development of panSarbecovirus NAb drugs.

Our study also offers important instructions for vaccine design. Vaccines are the most important tools for eventually vanquishing the SARS-CoV-2 pandemic. Based on our findings, we hypothesize that a vaccination strategy that helps to enrich Groups E and F NAbs would be promising to achieve broad protection. Along this line, Tan et al. recently showed that high-level and broadspectrum NAbs are present in the SARS-CoV convalescents who have received an mRNA vaccine based on the SARS-CoV-2 Spike ${ }^{52}$. We suspect that Groups E and F NAbs existed in SARS-CoV convalescents and might have been boosted upon SARS-CoV-2 vaccination. Because Groups E and F NAbs are the only effective NAbs against Omicron, the vaccines preferentially stimulating these antibodies are highly desired. Future studies are required to investigate a potential serial immunization scheme involving different 
inactivated Sarbecoviruses or their RBDs is capable of broadly protecting from future SARS-CoV-2 VOCs and even future Sarbecoviruses.

\section{Acknowledgments}

We thank Professor Jesse Bloom for his generous gift of the yeast SARS-CoV2 RBD libraries. We thank Beijing BerryGenomics for the help on DNA sequencing. We thank Sino Biological Inc. for the technical assistance on mAbs and B.1.1.529 RBD expression. This project is financially supported by the Ministry of Science and Technology of China (CPL-1233).

\section{Author contributions}

Y.C. and X.S.X designed the study. Y.C. and F.S coordinated the characterizations of the NAbs. J.W., F.J., H.L., H.S. performed and analyzed the yeast display mutation screening experiments. T.X., W.J., X.Y., P.W., H.L. performed the pseudovirus neutralization assays. W.H., Q.L., T.L., Y.Y., Q.C., S.L., Y.W. prepared the VSV-based SARS-CoV-2 pseudovirus. A.Y., Y.W., S.Y., R.A., W.S. performed and analyzed the antigen-specific single B cell VDJ sequencing. X.N., R.A. performed the antibody BLI studies. Z.C., S.D., P.L., L.W., Z.Z., X.W., J.X. performed the antibody structural analyses. P.W., Y.W., J.W, H.S, H.L. performed ELISA experiment. X.H. and R.J. coordinated the blood samples of vaccinated SARS convalescents. Y.C., X.W., J.X., X.S.X wrote the manuscript with inputs from all authors.

\section{Declaration of interests}

X.S.X. and Y.C. are inventors on the patent application of DXP-604 and BD series antibodies. X.S.X. and Y.C. are founders of Singlomics Biopharmaceuticals Inc. Other authors declare no competing interests.

\section{Methods}

\section{Antigen-specific B cell sorting and sequencing}

PBMCs were separated from whole blood samples based on the detailed protocol as described previously ${ }^{12}$. Briefly, blood samples were first diluted with 2\% FBS (Gibco) in PBS (Invitrogen) and subjected to Ficoll (Cytiva) gradient 
centrifugation. After red blood cell lysis and washing steps, PBMCs were resuspended with $2 \%$ FBS in PBS for downstream B cell isolation or $10 \%$ DMSO (Sigma-Aldrich) in FBS for further preservation.

Starting with freshly isolated or thawed PBMCs, B cells were enriched by positive selection using a CD19+ B cell isolation kit according to the manufacturer's instructions (STEMCELL). The enriched $B$ cells were stained in FACS buffer (1× PBS, 2\% FBS, 1 mM EDTA) with the following anti-human antibodies and antigens: FITC anti-CD19 Antibody (Biolegend), FITC antiCD20 Antibody (Biolegend), Brilliant Violet 421 anti-CD27 Antibody (Biolegend), PE/Cyanine7 anti-lgM, and fluorophore-labelled RBD (SARSCoV-2 and SARS-CoV RBD, Sino Biological Inc.) and ovalbumin (Ova) for 30 min on ice. Cells were stained with 7-AAD for 10 minutes before sorting. Single CD19 or CD20+ CD27+ IgM- Ova- RBD-PE+ RBD-APC+B cells were sorted on an Astrios EQ (BeckMan Coulter) into PBS containing 30\% FBS. Cells obtained after FACS were proceed to 5 '-mRNA and V(D)J libraries preparation as previously described ${ }^{12}$, which were further submitted to illumine sequencing on a Hiseq 2500 platform, with the 26x91 pair-end reading mode.

\section{V(D)J sequence data analysis}

The raw FASTQ files were processed by Cell Ranger (version 6.1.1) pipeline using GRCh38 reference. Sequences were generated using "cellranger multi" or "cellranger vdj" with default parameters. Then we extracted the protein sequences and processed them by IMGT/DomainGapAlign (version 4.10.2) to obtain the annotations of $V(D) J$, regions of $C D R$ and the mutation frequency ${ }^{53,54}$. Mutation count divided by the length of $V$ gene peptide is defined as the amino acid mutation rate of $\mathrm{V}$ gene.

\section{Recombinant antibody production}

Paired immunoglobulin heavy and light chain genes obtained from 10X Genomics V(D)J sequencing and analysis were submitted to recombinant monoclonal antibody synthesis. Briefly, heavy and light genes were cloned into expression vectors respectively based on Gibson assembly, which were 
subsequently co-transfected into HEK293 cells. Then secreted monoclonal antibodies from cultured cell were purified by protein A affinity chromatography. The specificities of these antibodies were determined by ELISA binding analysis.

\section{ELISA and neutralization assay}

ELISA were conducted to evaluate antibody or plasma binding ability. Briefly, after coating with $0.03 \mu \mathrm{g} / \mathrm{mL}$ and $1 \mu \mathrm{g} / \mathrm{mL}$ RBD (Sino Biological Inc.) of different sarbecovirus and SARS-CoV-2 variants, blocking, and washing, $1 \mu \mathrm{g} / \mathrm{mL}$ antibodies or serially diluted plasma samples were added to the plates. After incubation and wash, plates were incubated with diluted goat anti-human IgG $(\mathrm{H}+\mathrm{L}) / \mathrm{HRP}$ (JACKSON). Then plates were developed by addition of the TMB (Solarbio), then the developing reaction was stopped by adding $\mathrm{H}_{2} \mathrm{SO}_{4}$ and OD450 was measured by a ELISA microplate reader.

Neutralization assay was performed to evaluate neutralizing ability of antibody and plasma the detailed process was previously described by Cao et al. ${ }^{12}$. Briefly, serially diluted antibodies were first incubated with pseudotyped virus for $1 \mathrm{~h}$, and the mixture was then incubated with Huh-7 cells. After $24 \mathrm{~h}$ incubation in an incubator at $37 \mathrm{C}^{\circ}$, cells were collected and lysed with luciferase substrate (PerkinElmer), then proceed to luminescence intensity measurement by a microplate reader. IC50 and NT50 were determined by a four-parameter non-linear regression model. Omicron pseudovirus contains the following mutations: A67V, H69del, V70del, T95I, G142D, V143del, Y144del, Y145del, N211del, L212I, ins214EPE, G339D, S371L, S373P, S375F, K417N, N440K, G446S, S477N, T478K, E484A, Q493R, G496S, Q498R, N501Y, Y505H, T547K, D614G, H655Y, N679K, P681H, N764K, D796Y, N856K, Q954H, N969K, L981F.

\section{Biolayer interferometry}

Biolayer interferometry assays were conducted on Octet $₫$ R8 Protein Analysis System (Fortebio) following the manufacturer's instruction. Briefly, after baseline calibration, Protein A biosensors (Fortebio) were immersed with antibodies to capture the antibody, then sensors were immersed in PBS with 
$0.05 \%$ Tween-20 to the baseline. After association with different concentrations of RBD or Spike of Sarbecovirus and SARS-CoV-2 variants (Omicron RBD: 40592-V08H85, Omicron Spike: 40589-V08H26), disassociation was conducted. Data were recorded using software Data Acquisition 11.1 (Fortebio) and analyzed using software Data Analysis HT 11.1 (Fortebio).

\section{RBD Deep Mutational Scanning Library construction}

The yeast-display RBD mutant libraries used here were constructed as described by Starr et al., ${ }^{13}$ based on the spike receptor binding domain (RBD) from SARS-CoV-2 (NCBI GenBank: MN908947, residues N331-T531) with the modifications that instead of 16-neuclotide barcode, a unique 26-neuclotide barcode was appended to each RBD variant as an identifier in order to decrease sequencing cost by eliminating the use of PhiX. Briefly, three rounds of mutagenesis PCR were performed with designed and synthesized mutagenetic primer pools; in order to solid our conclusion, we constructed two RBD mutant libraries independently. RBD mutant libraries were then cloned into pETcon 2649 vector and the assembled products were electroporated into electrocompetent $\mathrm{DH} 10 \mathrm{~B}$ cells to enlarge plasmid yield. Plasmid extracted form E. coli were transformed into the EBY100 strain of Saccharomyces cerevisiae via the method described by Gietz and Schiest| ${ }^{55}$. Transformed yeast population were screened on SD-CAA selective plate and further cultured in SD-CAA liquid medium at a large scale. The resulted yeast libraries were flash frozen by liquid nitrogen and preserved at $-80 \mathrm{C}^{\circ}$.

\section{PacBio library preparation, sequencing and analysis}

The correspondence of RBD gene sequence in mutant library and N26 barcode was obtained by PacBio sequencing. Firstly, the bacterially-extracted plasmid pools were digested by Notl restriction enzyme and purified by agarose gel electrophoresis, then proceed to SMRTbell ligation. Four RBD mutant libraries were sequenced in one SMRT cell on a PacBio Sequel II platform. PacBio SMRT sequencing subreads were converted to HiFi ccs reads with pbccs, and then processed with a slightly modified version of the script previously 
described $^{13}$ to generate the barcode-variant dictionary. To reduce noise, variants containing stop codons or supported by only one ccs read were removed from the dictionary and ignored during further analysis.

\section{MACS-based mutation escape profiling}

ACE2 binding mutants were sorted based on magnetic beads to eliminate nonfunctional RBD variants. Briefly, the biotin binder beads (Thermo Fisher) were washed and prepared as the manufacturer's instruction and incubated with biotinylated ACE2 protein (Sino Biological Inc.) at room temperature with mild rotation. The ACE2 bound beads were washed twice and resuspend with $0.1 \%$ BSA buffer (PBS supplemented with $0.1 \%$ bovine serum albumin), and ready for ACE2 positive selection. Transformed yeast library were inoculated into SDCAA and grown at $30 \mathrm{C}^{\circ}$ with shaking for $16-18 \mathrm{~h}$, then back-diluted into SG$\mathrm{CAA}$ at $23 \mathrm{C}^{\circ}$ with shaking to induce RBD surface expression. Yeasts were collected and washed twice with $0.1 \%$ BSA buffer and incubated with aforementioned ACE2 bound beads at room temperature for $30 \mathrm{~min}$ with mild rotating. Then, the bead-bound cells were washed, resuspend with SD-CAA media, and grown at $30 \mathrm{C}^{\circ}$ with shaking. After overnight growth, the beadunbound yeasts were separated with a magnet and cultured in a large scale. The above ACE2 positive selected yeast libraries were preserved at $-80 C^{\circ}$ in aliquots as a seed bank for antibody escape mapping.

One aliquot of ACE2 positive selected RBD library was thawed and inoculated into SD-CAA, then grown at $30 \mathrm{C}^{\circ}$ with shaking for $16-18 \mathrm{~h} .120 \mathrm{OD}$ units were back-diluted into SG-CAA media and induced for RBD surface expression. Two rounds of sequential negative selection to sort yeast cells that escape Protein A conjugated antibody binding were performed according to the manufacturer's protocol. Briefly, Protein A magnetic beads (Thermo Fisher) were washed and resuspend in PBST (PBS with 0.02\% Tween-20). Then beads were incubated with neutralizing antibody and rotated at room temperature for $30 \mathrm{~min}$. The antibody-conjugated beads were washed and resuspend in PBST. Induced yeast libraries were washed and incubated with antibody-conjugated beads for $30 \mathrm{~min}$ at room temperature with agitation. The 
supernatant was separated and proceed to a second round of negative selection to ensure full depletion of antibody-binding yeast.

To eliminate yeast that did not express RBD, MYC-tag based RBD positive selection was conducted according to the manufacturer's protocol. First, anti-cMyc magnetic beads (Thermo Fisher) were washed and resuspend with $1 \mathrm{X}$ TBST, then the prepared beads were incubated for $30 \mathrm{~min}$ with the antibody escaping yeasts after two rounds of negative selection. Yeasts bound by antic-Myc magnetic beads were wash with 1X TBST and grown overnight in SDCAA to expand yeast population prior to plasmid extraction.

Overnight cultures of MACS sorted antibody-escaped and ACE2 preselected yeast populations were proceed to yeast plasmid extraction kit (Zymo Research). PCRs were performed to amplify the N26 barcode sequences as previously described ${ }^{13}$. The PCR products were purified with 0.9X Ampure XP beads (Beckman Coulter) and submitted to $75 \mathrm{bp}$ single-end Illumina Nextseq 500 sequencing.

\section{Deep mutational scanning data processing}

Raw single-end Illumina sequencing reads were trimmed and aligned to the reference barcode-variant dictionary generated as described above to get the count of each variant with dms_variants Python package (version 0.8.9). For libraries with N26 barcodes, we slightly modified the illuminabarcodeparser class of this package to tolerate one low sequencing quality base in the barcode region. The escape score of variant $\mathrm{X}$ is defined as $\mathrm{F} \times(\mathrm{nX}, \mathrm{ab} / \mathrm{Nab})$ / ( $\mathrm{nX}$, ref / Nref), where $n X, a b$ and $n X$,ref is the number of detected barcodes for variant $\mathrm{X}, \mathrm{Nab}$ and Nref are the total number of barcodes in antibody-selected (ab) library and reference (ref) library respectively as described by Starr et al. ${ }^{13}$. Different from FACS experiments, as we couldn't measure the number of cells retained after MACS selection precisely, here $F$ is considered as a scaling factor to transform raw escape fraction ratios to 0-1 range, and is calculated from the first and 99th percentiles of raw escape fraction ratios. Scores less than the first percentile or larger than the 99th percentile are considered to be 
outliers and set to zero or one, respectively. For each experiment, barcodes detected by $<6$ reads in the reference library were removed to reduce the impact of sampling noise, and variants with ACE2 binding below -2.35 or RBD expression below -1 were removed as previously described ${ }^{13}$. Finally, we built global epistasis models with dms_variants package for each library to estimate single mutation escape scores, utilizing the Python scripts provided by Greaney et al. ${ }^{18}$.

\section{Antibody clustering}

Antibody clustering and epitope group identification were performed based on the $\mathrm{N} \times \mathrm{M}$ escape score matrix, where $\mathrm{N}$ is the number of antibodies which pass the quality controlling filters, and $M$ is the number of informative sites on SARSCoV-2 RBD. Each entry of the matrix Anm refers to the total escape score of all kinds of mutations on site $\mathrm{m}$ of antibody $\mathrm{n}$. The dissimilarity between two antibodies is defined based on the Pearson's correlation coefficient of their escape score vectors, i. e. $\mathrm{Dij}=1-\operatorname{Corr}(\mathrm{Ai}, \mathrm{Aj})$. Sites with at least 6 escaped antibodies (site escape score $>1$ ) were considered informative and selected for dimensionality reduction and clustering. We utilized cmdscale $R$ function to convert the cleaned escape matrix into an $N \times 6$ feature matrix by multidimensional scaling (MDS) with the dissimilarity metric described above, followed by unsupervised k-medoids clustering within this 6-dimensional antibody feature space. Finally, two-dimensional tSNE embeddings were generated with Rtsne package for visualization. 
5201 Starr, T. N. et al. Prospective mapping of viral mutations that escape antibodies used to treat

2 Starr, T. N., Greaney, A. J., Dingens, A. S. \& Bloom, J. D. Complete map of SARS-CoV-2 RBD mutations that escape the monoclonal antibody LY-CoV555 and its cocktail with LY-CoV016. Cell Rep Med 2, 100255, doi:10.1016/j.xcrm.2021.100255 (2021).

3 Barnes, C. O. et al. SARS-CoV-2 neutralizing antibody structures inform therapeutic strategies. Nature 588, 682-687, doi:10.1038/s41586-020-2852-1 (2020).

4 Yuan, M. et al. Structural and functional ramifications of antigenic drift in recent SARS-CoV-2 variants. Science 373, 818-823, doi:10.1126/science.abh1139 (2021).

5 Dejnirattisai, W. et al. The antigenic anatomy of SARS-CoV-2 receptor binding domain. Cell 184, 2183-2200 e2122, doi:10.1016/j.cell.2021.02.032 (2021).

6 Pinto, D. et al. Cross-neutralization of SARS-CoV-2 by a human monoclonal SARS-CoV antibody. Nature 583, 290-295, doi:10.1038/s41586-020-2349-y (2020).

7 Yuan, M. et al. A highly conserved cryptic epitope in the receptor binding domains of SARSCoV-2 and SARS-CoV. Science 368, 630-633, doi:10.1126/science.abb7269 (2020).

8 Callaway, E. Heavily mutated Omicron variant puts scientists on alert. Nature 600, 21, doi:10.1038/d41586-021-03552-w (2021).

9 Callaway, E. \& Ledford, H. How bad is Omicron? What scientists know so far. Nature, doi:10.1038/d41586-021-03614-z (2021).

10 Kumar, S., Thambiraja, T. S., Karuppanan, K. \& Subramaniam, G. Omicron and Delta Variant of SARS-CoV-2: A Comparative Computational Study of Spike protein. bioRxiv (2021).

11 Li, Q. et al. SARS-CoV-2 501Y.V2 variants lack higher infectivity but do have immune escape. Cell 184, 2362-2371 e2369, doi:10.1016/j.cell.2021.02.042 (2021).

12 Cao, Y. et al. Humoral immune response to circulating SARS-CoV-2 variants elicited by inactivated and RBD-subunit vaccines. Cell Res 31, 732-741, doi:10.1038/s41422-021-00514-9 (2021).

13 Starr, T. N. et al. Deep Mutational Scanning of SARS-CoV-2 Receptor Binding Domain Reveals Constraints on Folding and ACE2 Binding. Cell 182, 1295-1310 e1220, doi:10.1016/j.cell.2020.08.012 (2020).

$14 \mathrm{Gu}, \mathrm{H}$. et al. Adaptation of SARS-CoV-2 in BALB/c mice for testing vaccine efficacy. Science 369, 1603-1607, doi:10.1126/science.abc4730 (2020).

15 Cerutti, G. et al. Potent SARS-CoV-2 neutralizing antibodies directed against spike N-terminal domain target a single supersite. Cell Host Microbe 29, 819-833 e817, doi:10.1016/j.chom.2021.03.005 (2021).

16 McCallum, M. et al. N-terminal domain antigenic mapping reveals a site of vulnerability for SARS-CoV-2. Cell 184, 2332-2347 e2316, doi:10.1016/j.cell.2021.03.028 (2021).

17 Yuan, M., Liu, H., Wu, N. C. \& Wilson, I. A. Recognition of the SARS-CoV-2 receptor binding domain by neutralizing antibodies. Biochem Biophys Res Commun 538, 192-203, doi:10.1016/j.bbrc.2020.10.012 (2021).

18 Greaney, A. J. et al. Complete Mapping of Mutations to the SARS-CoV-2 Spike ReceptorBinding Domain that Escape Antibody Recognition. Cell Host Microbe 29, 44-57 e49, doi:10.1016/j.chom.2020.11.007 (2021).

19 Cao, Y. et al. Potent Neutralizing Antibodies against SARS-CoV-2 Identified by HighThroughput Single-Cell Sequencing of Convalescent Patients' B Cells. Cell 182, 73-84 e16, doi:10.1016/j.cell.2020.05.025 (2020).

20 Ju, B. et al. Human neutralizing antibodies elicited by SARS-CoV-2 infection. Nature 584, $115-$ 119, doi:10.1038/s41586-020-2380-z (2020).

21 Shi, R. et al. A human neutralizing antibody targets the receptor-binding site of SARS-CoV-2. Nature 584, 120-124, doi:10.1038/s41586-020-2381-y (2020).

$22 \mathrm{Wu}$, Y. et al. A noncompeting pair of human neutralizing antibodies block COVID-19 virus binding to its receptor ACE2. Science 368, 1274-1278, doi:10.1126/science.abc2241 (2020).

23 Yuan, M. et al. Structural basis of a shared antibody response to SARS-CoV-2. Science 369, 1119-1123, doi:10.1126/science.abd2321 (2020). 
24 Robbiani, D. F. et al. Convergent antibody responses to SARS-CoV-2 in convalescent individuals. Nature 584, 437-442, doi:10.1038/s41586-020-2456-9 (2020).

25 Liu, L. et al. Potent neutralizing antibodies against multiple epitopes on SARS-CoV-2 spike. Nature 584, 450-456, doi:10.1038/s41586-020-2571-7 (2020).

26 Baum, A. et al. Antibody cocktail to SARS-CoV-2 spike protein prevents rapid mutational escape seen with individual antibodies. Science 369, 1014-1018, doi:10.1126/science.abd0831 (2020).

27 Brouwer, P. J. M. et al. Potent neutralizing antibodies from COVID-19 patients define multiple targets of vulnerability. Science 369, 643-650, doi:10.1126/science.abc5902 (2020).

28 Rogers, T. F. et al. Isolation of potent SARS-CoV-2 neutralizing antibodies and protection from disease in a small animal model. Science 369, 956-963, doi:10.1126/science.abc7520 (2020).

29 Piccoli, L. et al. Mapping Neutralizing and Immunodominant Sites on the SARS-CoV-2 Spike Receptor-Binding Domain by Structure-Guided High-Resolution Serology. Cell 183, 1024-1042 e1021, doi:10.1016/j.cell.2020.09.037 (2020).

30 Zost, S. J. et al. Potently neutralizing and protective human antibodies against SARS-CoV-2. Nature 584, 443-449, doi:10.1038/s41586-020-2548-6 (2020).

31 Tortorici, M. A. et al. Ultrapotent human antibodies protect against SARS-CoV-2 challenge via multiple mechanisms. Science 370, 950-957, doi:10.1126/science.abe3354 (2020).

32 Lv, Z. et al. Structural basis for neutralization of SARS-CoV-2 and SARS-CoV by a potent therapeutic antibody. Science 369, 1505-1509, doi:10.1126/science.abc5881 (2020).

33 Zost, S. J. et al. Rapid isolation and profiling of a diverse panel of human monoclonal antibodies targeting the SARS-CoV-2 spike protein. Nat Med 26, 1422-1427, doi:10.1038/s41591-020-0998-x (2020).

34 Seydoux, E. et al. Analysis of a SARS-CoV-2-Infected Individual Reveals Development of Potent Neutralizing Antibodies with Limited Somatic Mutation. Immunity 53, 98-105 e105, doi:10.1016/j.immuni.2020.06.001 (2020).

35 Kreye, J. et al. A Therapeutic Non-self-reactive SARS-CoV-2 Antibody Protects from Lung Pathology in a COVID-19 Hamster Model. Cell 183, 1058-1069 e1019, doi:10.1016/j.cell.2020.09.049 (2020).

36 Scheid, J. F. et al. B cell genomics behind cross-neutralization of SARS-CoV-2 variants and SARS-CoV. Cell 184, 3205-3221 e3224, doi:10.1016/j.cell.2021.04.032 (2021).

37 Tortorici, M. A. et al. Broad sarbecovirus neutralization by a human monoclonal antibody. Nature 597, 103-108, doi:10.1038/s41586-021-03817-4 (2021).

38 Dong, J. et al. Genetic and structural basis for SARS-CoV-2 variant neutralization by a twoantibody cocktail. Nat Microbiol 6, 1233-1244, doi:10.1038/s41564-021-00972-2 (2021).

39 Starr, T. N. et al. SARS-CoV-2 RBD antibodies that maximize breadth and resistance to escape. Nature 597, 97-102, doi:10.1038/s41586-021-03807-6 (2021).

40 Martinez, D. R. et al. A broadly cross-reactive antibody neutralizes and protects against sarbecovirus challenge in mice. Sci Transl Med, eabj7125, doi:10.1126/scitranslmed.abj7125 (2021).

41 Onodera, T. et al. A SARS-CoV-2 antibody broadly neutralizes SARS-related coronaviruses and variants by coordinated recognition of a virus-vulnerable site. Immunity 54, 2385-2398 e2310, doi:10.1016/j.immuni.2021.08.025 (2021).

42 Raybould, M. I. J., Kovaltsuk, A., Marks, C. \& Deane, C. M. CoV-AbDab: the coronavirus antibody database. Bioinformatics 37, 734-735, doi:10.1093/bioinformatics/btaa739 (2021).

43 Barnes, C. O. et al. Structures of Human Antibodies Bound to SARS-CoV-2 Spike Reveal Common Epitopes and Recurrent Features of Antibodies. Cell 182, 828-842 e816, doi:10.1016/j.cell.2020.06.025 (2020).

44 Hansen, J. et al. Studies in humanized mice and convalescent humans yield a SARS-CoV-2 antibody cocktail. Science 369, 1010-1014, doi:10.1126/science.abd0827 (2020).

45 Kim, S. I. et al. Stereotypic Neutralizing VH Clonotypes Against SARS-CoV-2 RBD in COVID-19 Patients and the Healthy Population. bioRxiv, 2020.2006.2026.174557, doi:10.1101/2020.06.26.174557 (2020).

46 Du, S. et al. Structurally Resolved SARS-CoV-2 Antibody Shows High Efficacy in Severely Infected Hamsters and Provides a Potent Cocktail Pairing Strategy. Cell 183, 1013-1023 e1013, doi:10.1016/j.cell.2020.09.035 (2020). 
Du, S. et al. Structures of SARS-CoV-2 B.1.351 neutralizing antibodies provide insights into cocktail design against concerning variants. Cell Res 31, 1130-1133, doi:10.1038/s41422-021-

48 Harvey, W. T. et al. SARS-CoV-2 variants, spike mutations and immune escape. Nat Rev Microbiol 19, 409-424, doi:10.1038/s41579-021-00573-0 (2021).

49 Jones, B. E. et al. The neutralizing antibody, LY-CoV555, protects against SARS-CoV-2 infection in nonhuman primates. Sci Transl Med 13, doi:10.1126/scitranslmed.abf1906 (2021).

50 Kemp, S. A. et al. SARS-CoV-2 evolution during treatment of chronic infection. Nature 592, 277-282, doi:10.1038/s41586-021-03291-y (2021).

51 Saito, A. et al. Enhanced fusogenicity and pathogenicity of SARS-CoV-2 Delta P681R mutation.

52 Tan, C. W. et al. Pan-Sarbecovirus Neutralizing Antibodies in BNT162b2-Immunized SARS-CoV1 Survivors. N Engl J Med 385, 1401-1406, doi:10.1056/NEJMoa2108453 (2021).

53 Ehrenmann, F., Kaas, Q. \& Lefranc, M. P. IMGT/3Dstructure-DB and IMGT/DomainGapAlign: a database and a tool for immunoglobulins or antibodies, T cell receptors, MHC, IgSF and MhcSF. Nucleic Acids Res 38, D301-307, doi:10.1093/nar/gkp946 (2010).

54 Ehrenmann, F. \& Lefranc, M. P. IMGT/DomainGapAlign: IMGT standardized analysis of amino acid sequences of variable, constant, and groove domains (IG, TR, MH, IgSF, MhSF). Cold Spring Harb Protoc 2011, 737-749, doi:10.1101/pdb.prot5636 (2011).

55 Gietz, R. D. \& Schiestl, R. H. High-efficiency yeast transformation using the LiAc/SS carrier DNA/PEG method. Nat Protoc 2, 31-34, doi:10.1038/nprot.2007.13 (2007). 

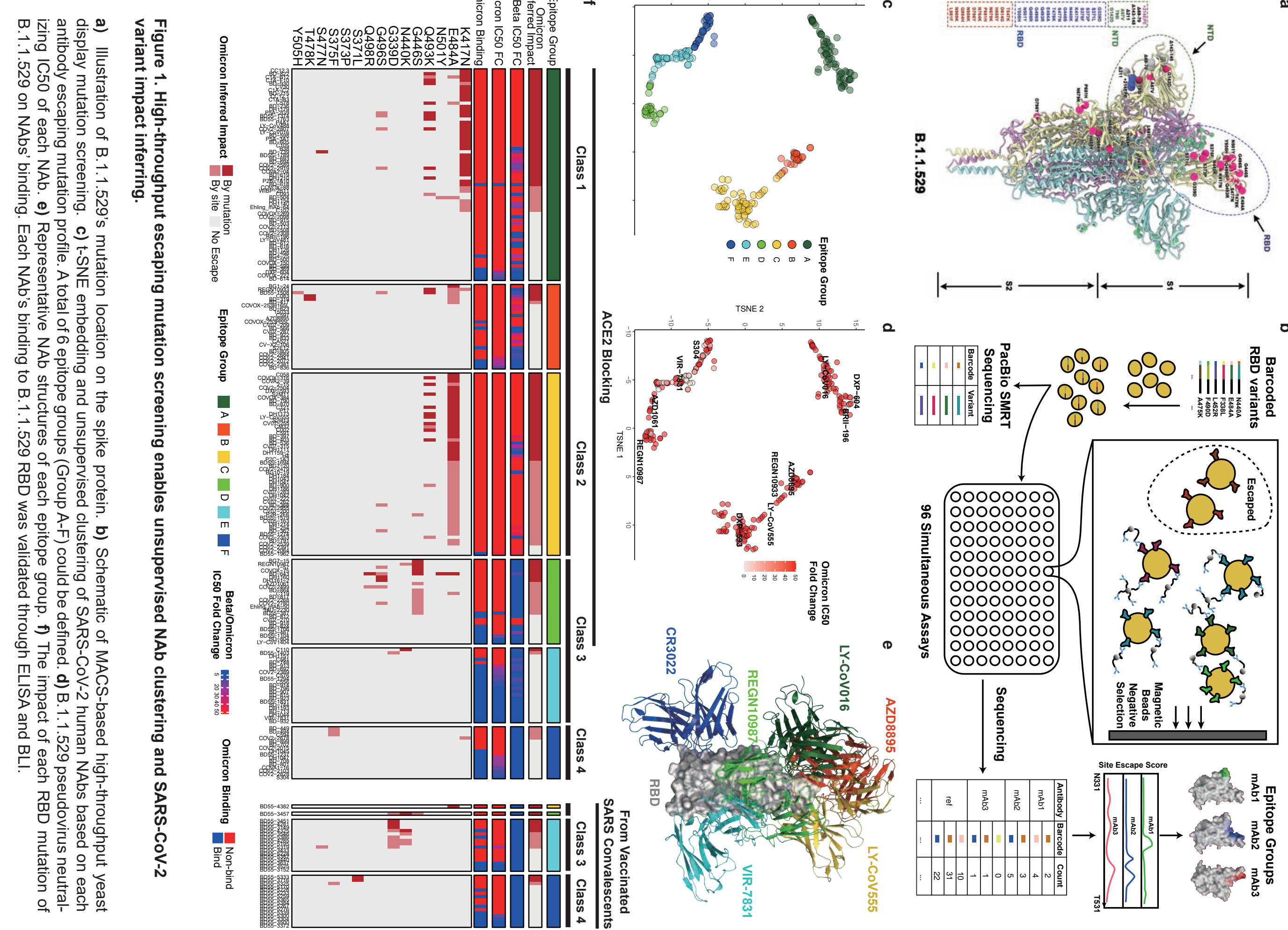


\section{a}

Epitope Group E

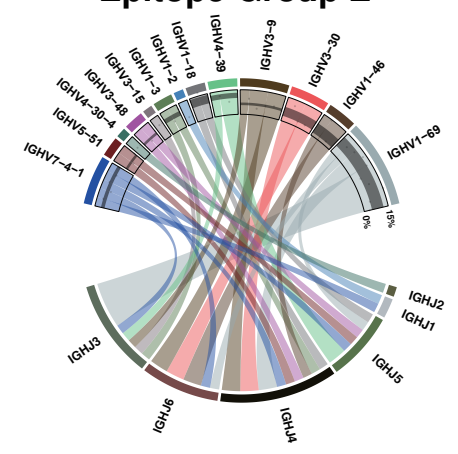

C.
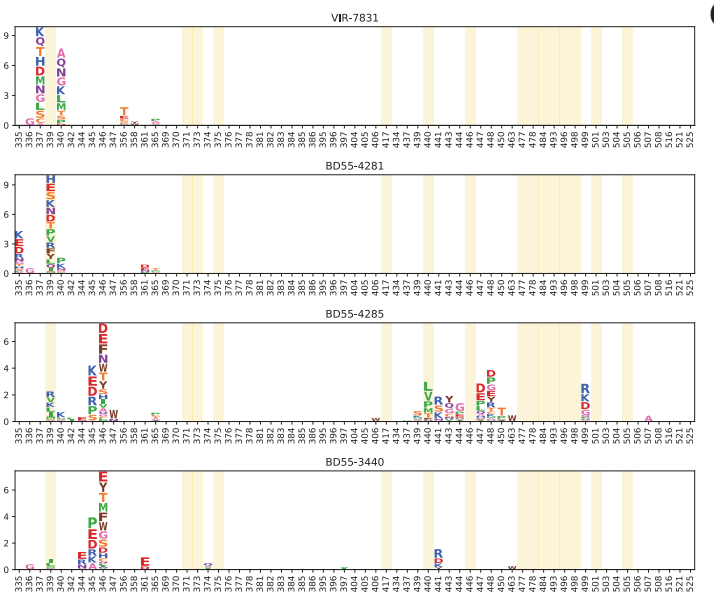

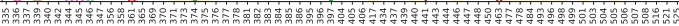

e

Site Escape Score
Omicron IC50
IC50 Fold Change $\begin{aligned} & 0.0010 .01 \\ & 5\end{aligned} 2030$

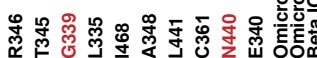

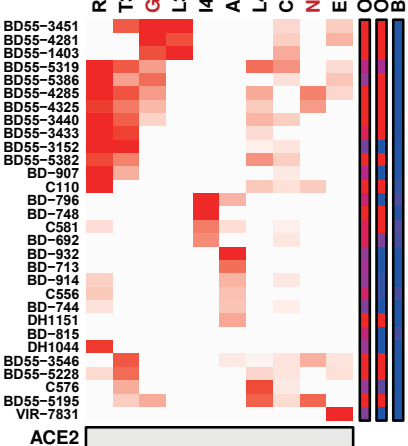

f

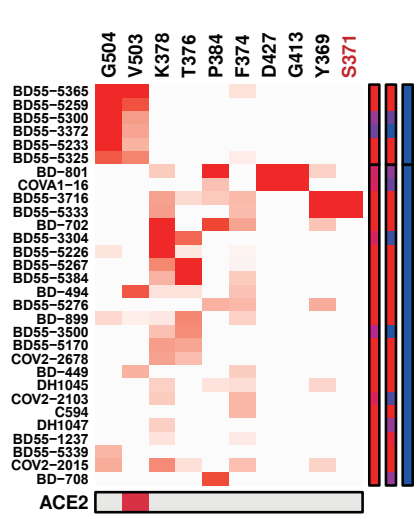

g

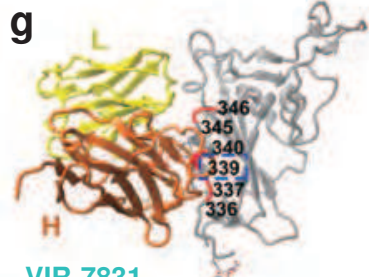

VIR-7831

h

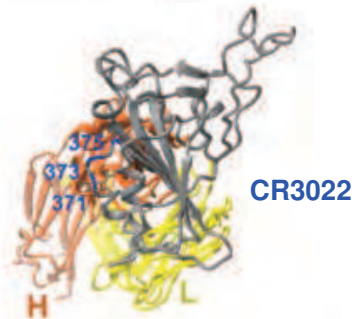

i
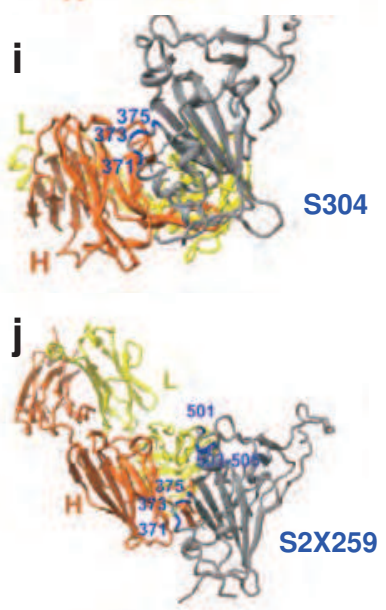

Figure 3. VDJ combination, escape maps, mutations of significant impact, and typical structures in complex with RBD for antibodies of epitope group E-F.

a-b VDJ combination of antibodies' heavy chain variable region and their mutation rate for group E-F, respectively. For each group, the upper semicircle represents IGHV gene distribution and the lower semicircles represent IGHJ gene distribution.

c-d Escape maps of represetative RBD NAbs for group E-F respectively. For each site, the height of a letter indicates the detected mutation escape score of its corresponding residue. Site mutated in Omicron (B.1.1.529) are highlighted.

e-f Heatmaps of site escape scores for RBD NAbs of epitope group E-F, respectively. ACE2 interface residues are annotated with red blocks, and mutated sites in Omicron are marked red. Annotates on the right side of heatmaps represent pseudovirus neutralizing IC50 fold change for Omicron and Beta in comparison with D614G.

g-j Typical structures in complex with RBD of group E-F antibodies. Residues involved in important contacts or related to Omicron mutations are marked. 
a
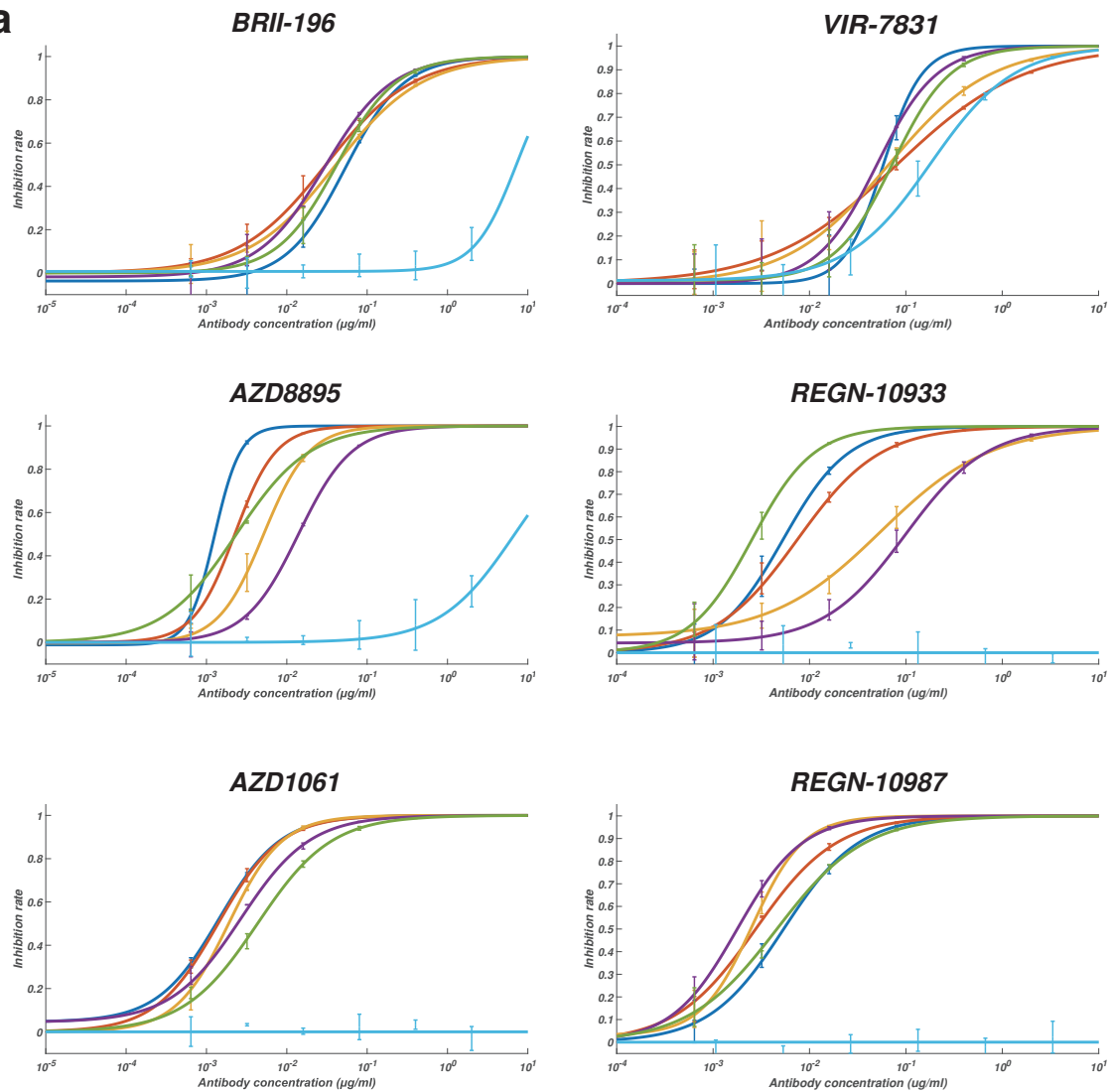

b

\begin{tabular}{|c|c|c|c|c|c|c|}
\hline 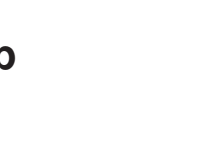 & 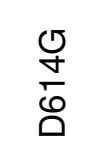 & $\frac{\text { 宅 }}{\frac{0}{2}}$ & $\begin{array}{l}\underset{\mathbb{E}}{E} \\
\underset{\mathbb{E}}{\mathbb{V}}\end{array}$ & $\begin{array}{l}\frac{\pi}{\Phi} \\
\mathbb{\infty}\end{array}$ & 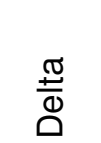 & $\begin{array}{c}\text { 든 } \\
\frac{0}{\delta} \\
\overline{0}\end{array}$ \\
\hline LY-CoV555 & 0.013 & 0.008 & $>10$ & $>10$ & $>10$ & $>10$ \\
\hline LY-CoV016 & 0.032 & 1.707 & $>10$ & $>10$ & 0.024 & $>10$ \\
\hline EGN10933 & 0.005 & 0.007 & 0.055 & 0.098 & 0.003 & $>10$ \\
\hline EGN10987 & 0.005 & 0.003 & 0.003 & 0.002 & 0.005 & $>10$ \\
\hline AZD8895 & 0.001 & 0.002 & 0.012 & 0.014 & 0.002 & 6.860 \\
\hline AZD1061 & 0.001 & 0.001 & 0.002 & 0.003 & 0.004 & $>10$ \\
\hline VIR-7831 & 0.058 & 0.080 & 0.066 & 0.050 & 0.073 & 0.181 \\
\hline BRII-196 & 0.053 & 0.031 & 0.041 & 0.030 & 0.042 & 7.258 \\
\hline DXP-604 & 0.010 & 0.007 & 0.005 & 0.065 & 0.016 & 0.287 \\
\hline & & & & & \multicolumn{2}{|c|}{ IC50 (ng/ $/ \mu \mathrm{L})$} \\
\hline
\end{tabular}
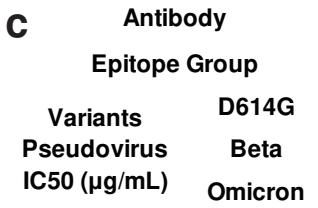

VIR-7831
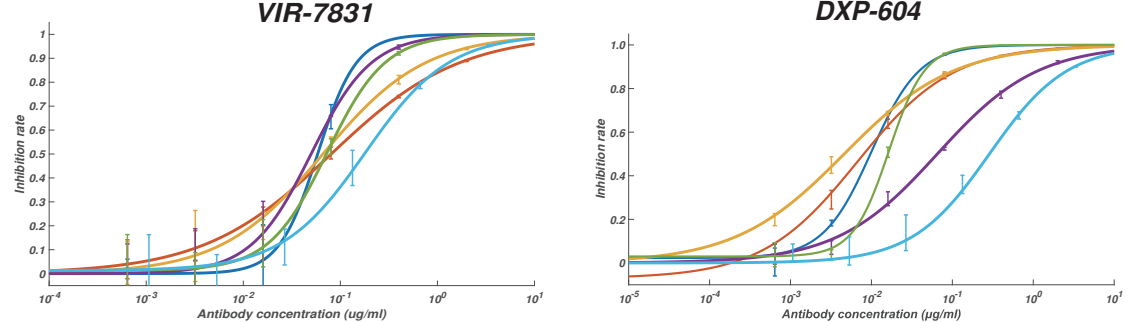

I $D 614 G$

I Alpha (B.1.1.7)

I Gamma (P.1)

I Beta (B.1.351)

I Delta (B.1.617.2)

I Omicron (B.1.1.529)

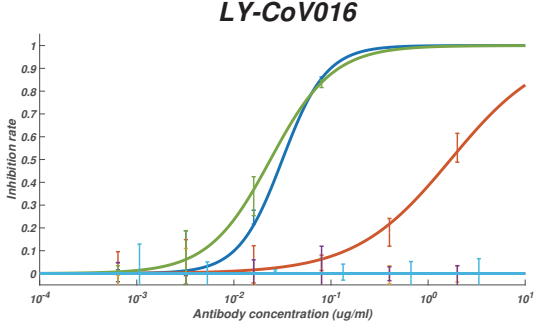

I D614G

I Alpha (B.1.1.7)

I Gamma (P.1)

I Beta (B.1.351)

I Delta (B.1.617.2)

I Omicron (B.1.1.529)

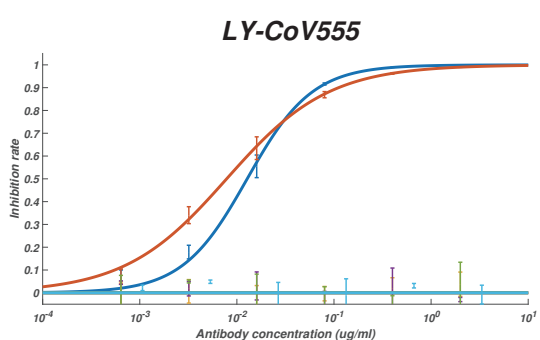

$\mp$ D614G
$\mp$ Alpha (B.1.1.7)
$\mp$ Gamma (P.1)
$\mp$ Beta (B.1.351)
$\mp$ Delta (B.1.617.2)
$\mp$ Omicron (B.1.1.529)

BD55-3152 BD55-5319 BD55-5386 BD55-5300 BD55-3372 BD55-3500

$\begin{array}{lllllll}E & \text { E } & \text { E } & F & F & F\end{array}$

$\begin{array}{llllll}0.0105 & 0.0150 & 0.0005 & 0.0051 & 0.0068 & 0.1053\end{array}$

$\begin{array}{llllll}0.0076 & 0.0040 & 0.0011 & 0.0031 & 0.0073 & 0.2326\end{array}$

$\begin{array}{llllll}0.0142 & 0.3685 & 0.0584 & 0.0663 & 0.0097 & 0.2610\end{array}$

\begin{tabular}{|c|c|c|c|c|c|c|c|}
\hline & SARS-CoV-1 & 3.462 & 3.447 & 3.417 & 3.405 & 3.467 & 3.476 \\
\hline \multirow{3}{*}{$\begin{array}{l}\stackrel{0}{0} \\
\frac{0}{0} \\
\frac{\pi}{0}\end{array}$} & RaTG13 & 0.353 & 0.135 & 0.086 & 0.193 & 0.061 & 3.258 \\
\hline & Pangolin-GD & 1.132 & 2.657 & 0.421 & 3.526 & 3.493 & 3.57 \\
\hline & Pangolin-GX & 0.996 & 0.812 & 1.95 & 0.709 & 2.889 & 3.403 \\
\hline \multirow{3}{*}{$\begin{array}{l}\frac{\pi}{0} \\
\frac{\pi}{0} \\
\frac{\pi}{0}\end{array}$} & $\begin{array}{c}\text { SARS-CoV-1 } \\
\text { PC4-127 }\end{array}$ & 3.598 & 3.523 & 3.473 & 3.531 & 3.557 & 3.457 \\
\hline & $\begin{array}{c}\text { SARS-CoV-1 } \\
\text { Sin852 }\end{array}$ & 3.458 & 3.495 & 3.468 & 3.464 & 3.413 & 2.984 \\
\hline & WIV1 & 2.409 & 1.976 & 3.336 & 3.588 & 3.515 & 3.603 \\
\hline กั Clade 3 & BM48-31 & 0.133 & 3.424 & 0.115 & 3.483 & 3.515 & 3.476 \\
\hline Clade 2 & YN2013 & 0.168 & 0.244 & 0.074 & 0.601 & 0.072 & 2.683 \\
\hline
\end{tabular}

Fig. 4 Pseudovirus neutralization and RBD binding affinity of various SARS-CoV-2 NAbs. a, Neutralization of SARS-CoV-2 variants of concern (pseudotyped VSV) by 9 clinical antibody drugs. Data are collected from three technical replicates, and error bars show mean \pm s.d. b, IC50 values calculated from data in Fig. 4a. c, SARS-CoV-2 variants pseudovirus IC50 and sarbecovirus RBD binding affinity (mea-sured by ELISA OD450) of selected potent Omicron-neutralizing antibodies. 

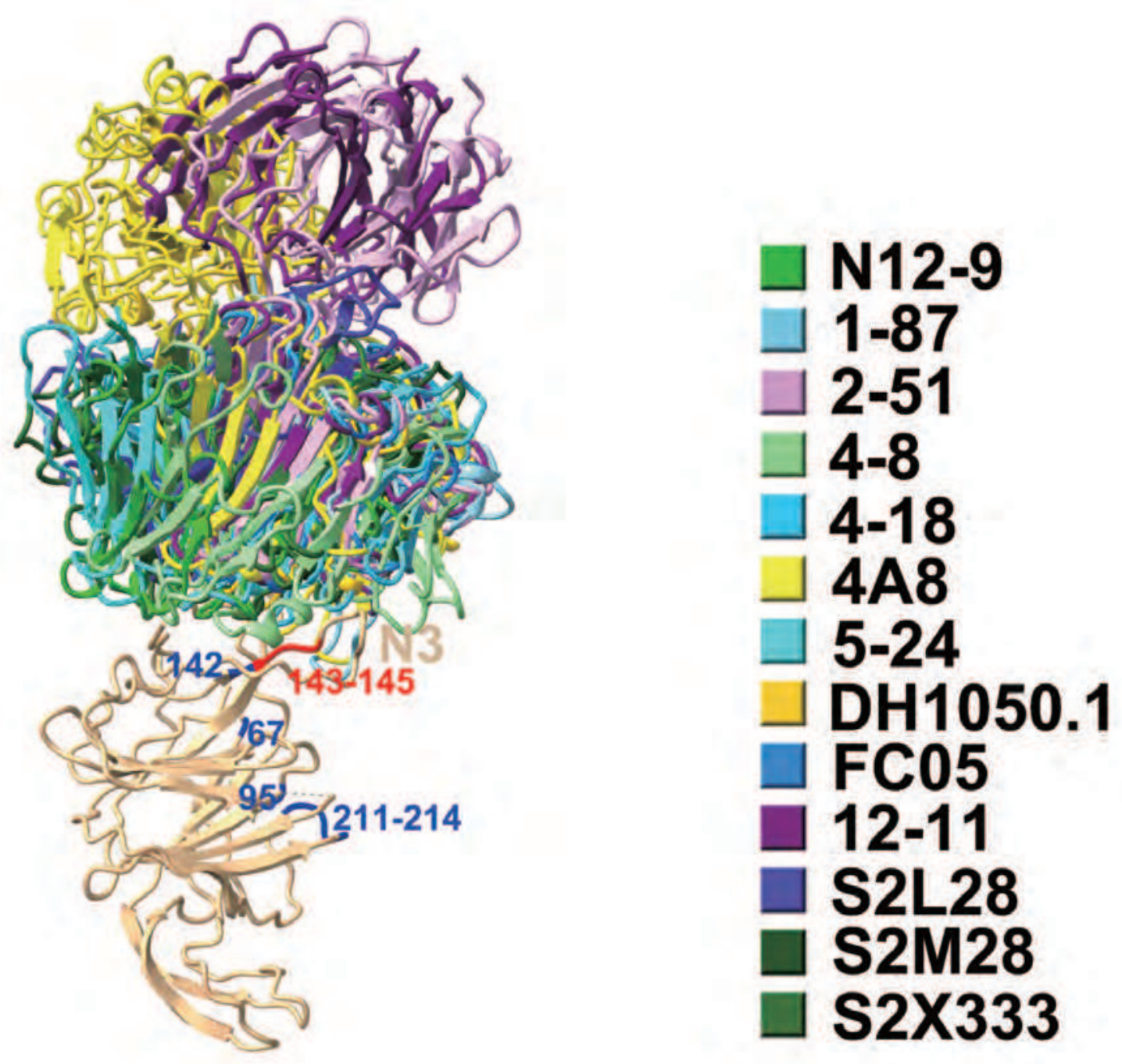

Extended Data Fig. 1 Structures of SARS-CoV-2 NTD-binding neutralizing antibodies.

NTD-binding NAbs are shown together in different colors in complex with one NTD, and most of them contact with residues 142-145, which indicates their high probability to be escaped by G142D and $\Delta$ 143-145 of Omicron. Missense mutations and deletions of Omicron NTD are colored blue and red, respectively. 


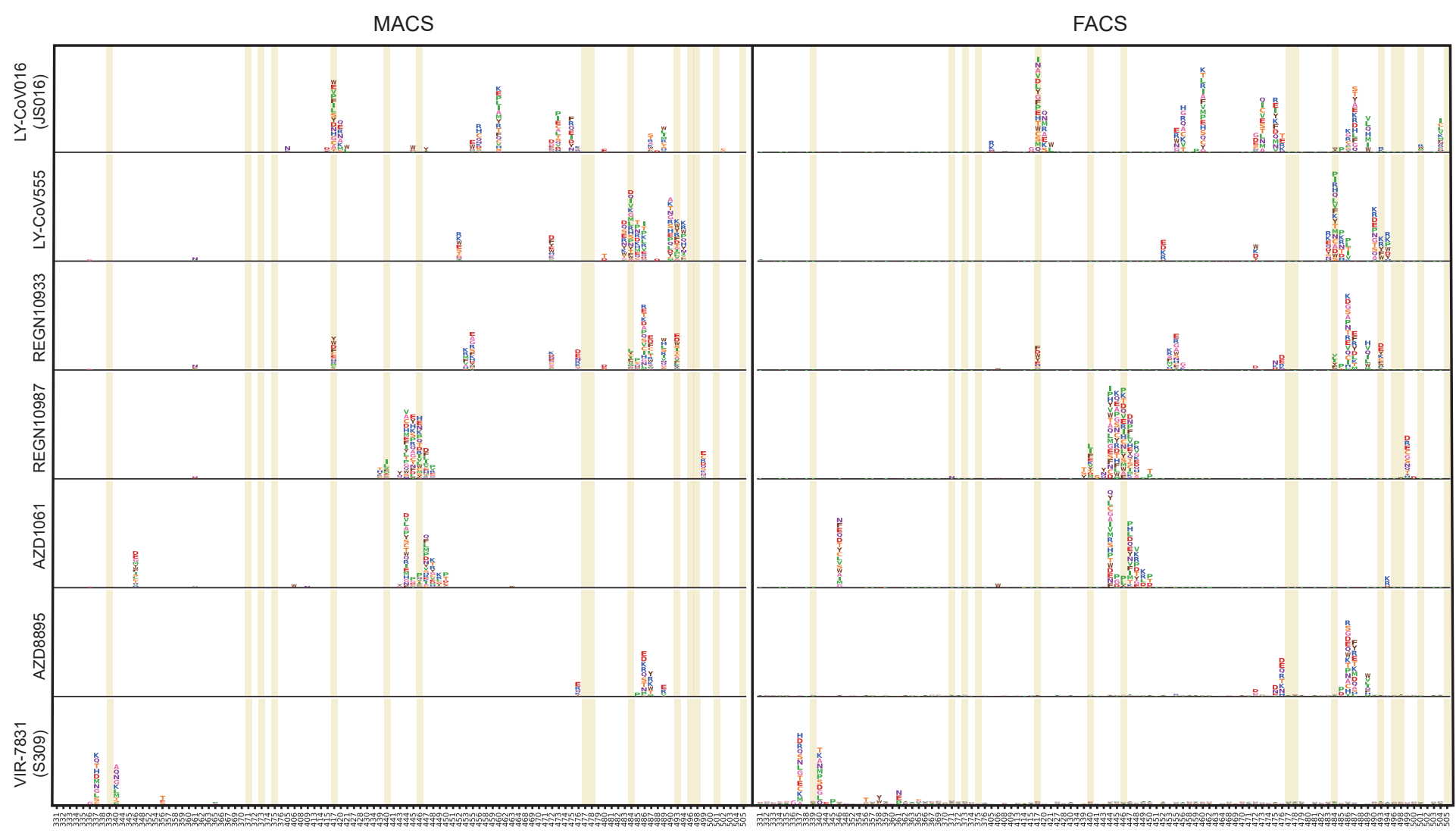

Extended Data Fig. 2 Comparison between FACS and MACS-based deep mutational scanning for epitope identification of SARS-CoV-2 RBD antibodies.

Deep mutational scanning maps with MACS-based (left) and FACS-based assays (right) of therapeutic neutralizing antibodies that have received emergency use authorization from the US FDA. Sites mutated in Omicron variant are highlighted. 

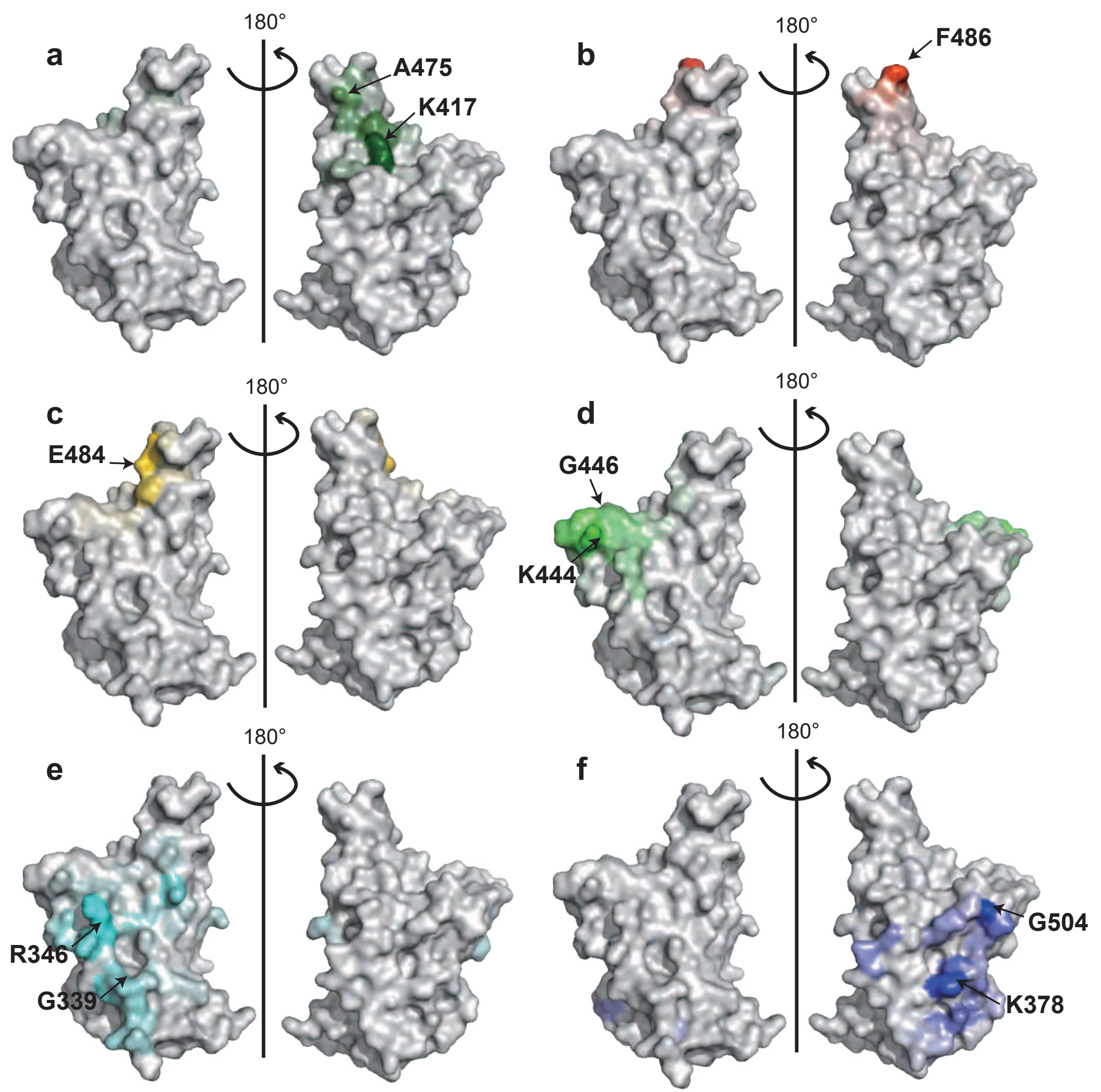

Extended Data Fig. 3 SARS-CoV-2 RBD antibodies of different epitope groups show distinct escape hotspots on RBD surface.

(a)-(f) correspond to aggregated site escape scores of epitope group A-F, respectively. Escape hotspots of each epitope group are annotated by arrows. 

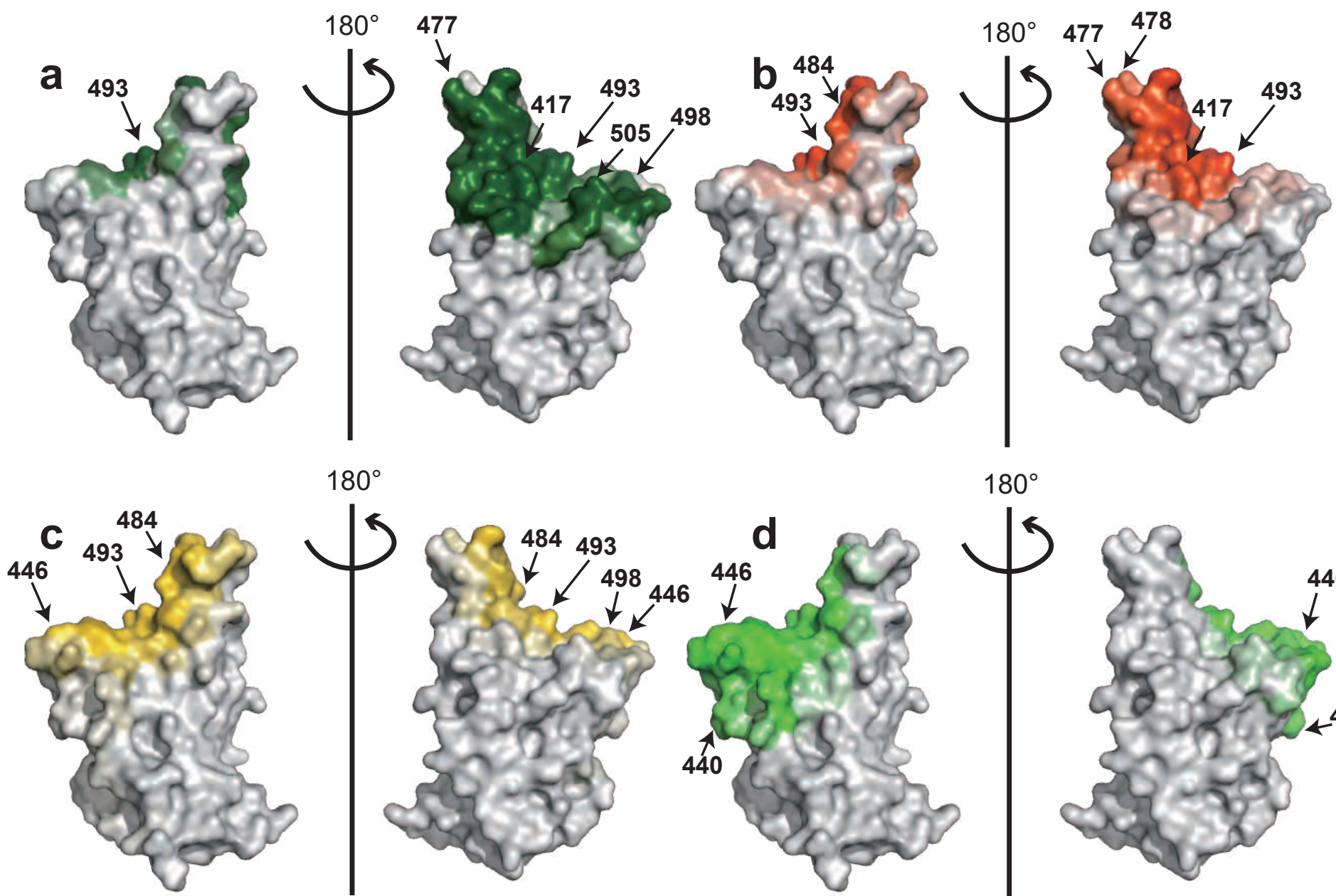

$180^{\circ}$
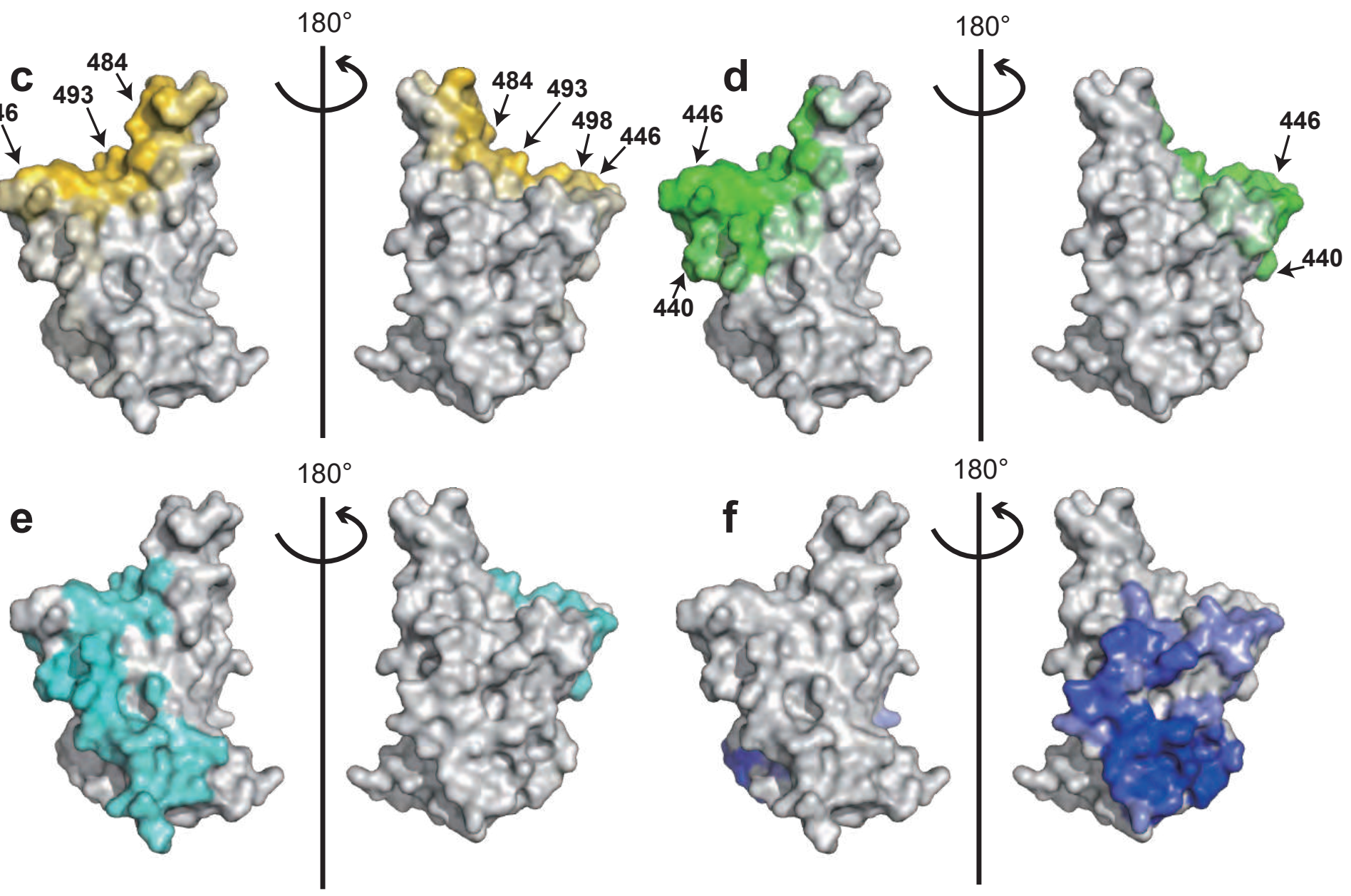

\section{Extended Data Fig. 4 SARS-CoV-2 RBD antibodies of different epitope groups show distinct} spatial contact footprints on RBD surface.

(a)-(f) correspond to aggregated footprints of epitope group A-F, respectively. Publicly availble structures of neutralizing antibodies in complex with SARS-CoV-2 RBD are gathered from PDB and classified into groups. Different colors distinguish epitope groups, and the darkness reflects group-specific site popularity to appear on the complex interface. Common interface residues of each group are marked by arrows. 
a

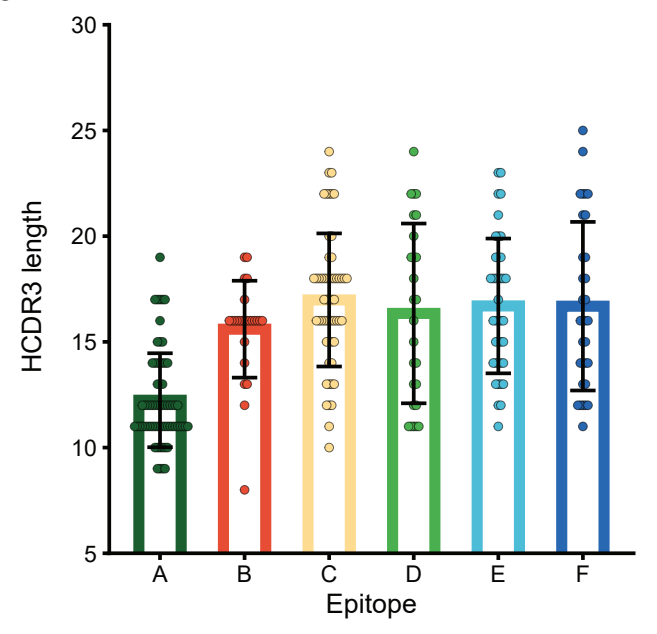

C

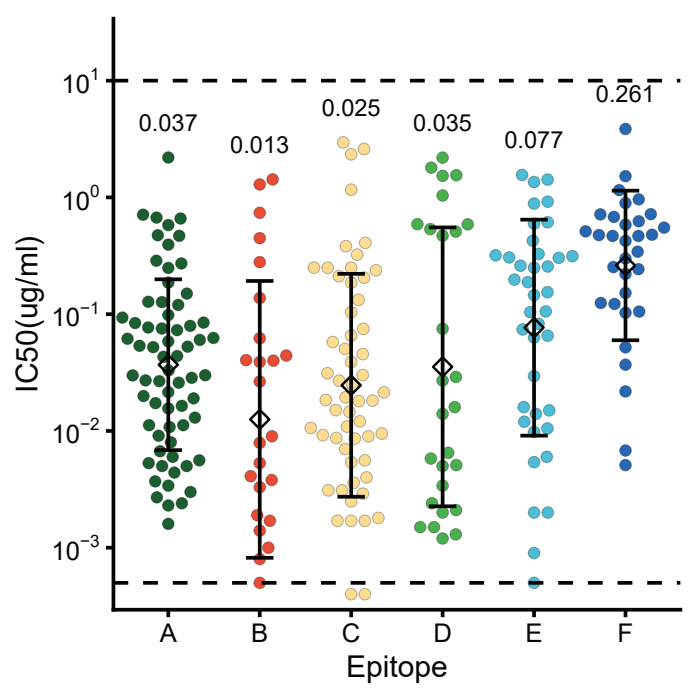

e

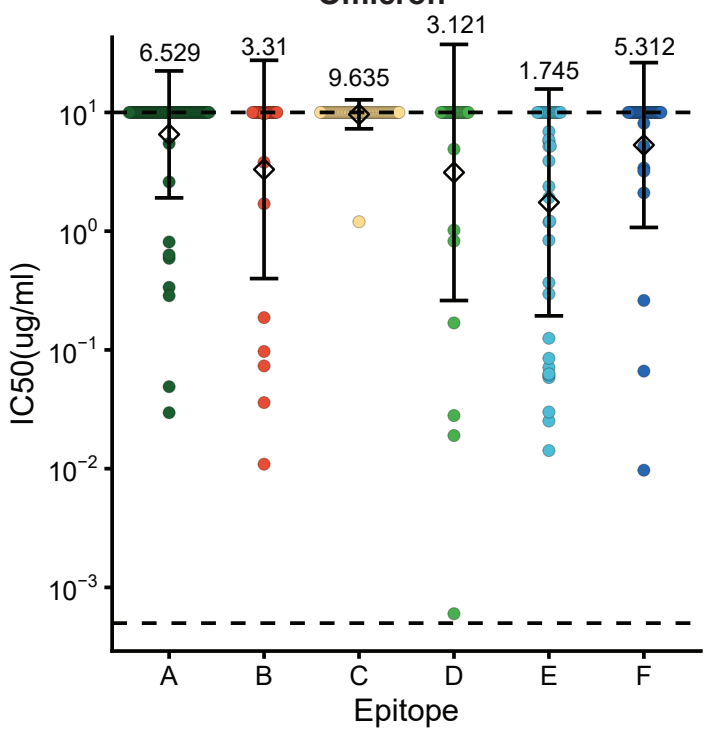

b

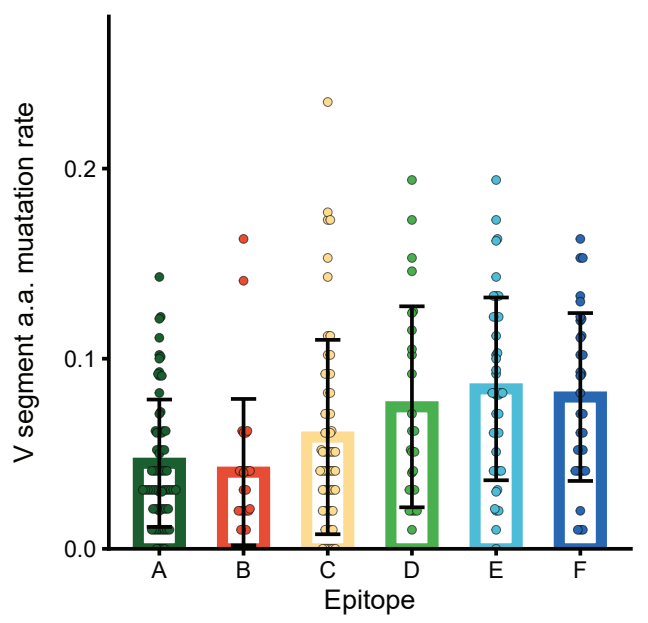

d

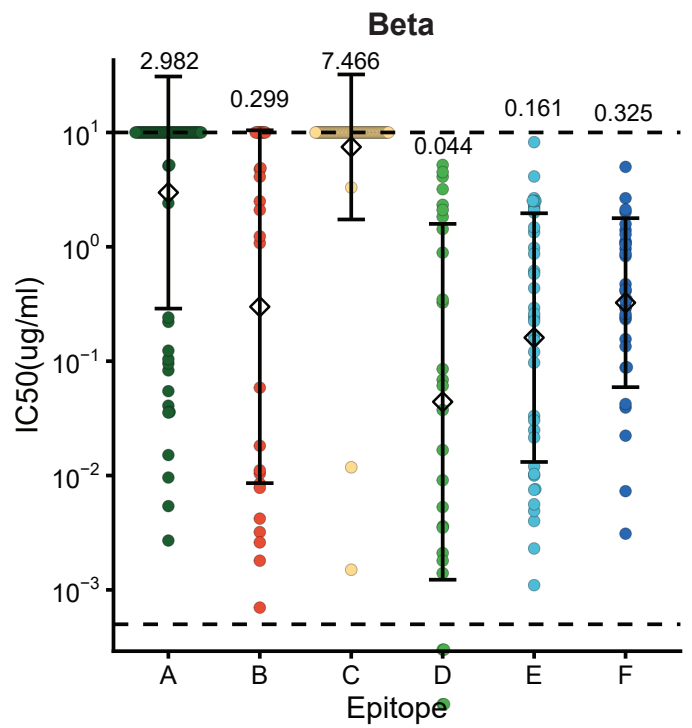

Extended Data Fig. 5. Antibody features and pseudovirus neutralizing IC50s.

a) The length of $\mathrm{H}$ chain complementarity-determining region 3(HCDR3) amino acid sequence. Scatters show the individual HCDR3 length of antibody, vertical bars show the mean value, and error bars show mean $\pm s d$. b) The $V$ segment amino acid(a.a.) mutation rate. Mutation count divided by the a.a. length of $\mathrm{V}$ segment is defined as the mutation rate of $\mathrm{V}$ segment. Scatters show the individual $\mathrm{H}$ chain $\mathrm{V}$ segment mutation rate of antibody, vertical bars show the mean value, and error bars show mean \pm sd.

c-e) IC50 of antibodies in the D614G(c), Beta(d), and Omicron(e) mutant pseudovirus neutralizing assay. Scatters show the individual IC50 of antibody, diamonds show the IC50 geometric mean(GM) of each epitope, and error bars show $\mathrm{GM} \pm \mathrm{sd}$ in the log10 scale. Dotted lines show the limit of detection, which is from $0.0005 \mathrm{ug} / \mathrm{ml}$ to $10 \mathrm{ug} / \mathrm{ml}$. IC50 GMs are also noted on the figure. 

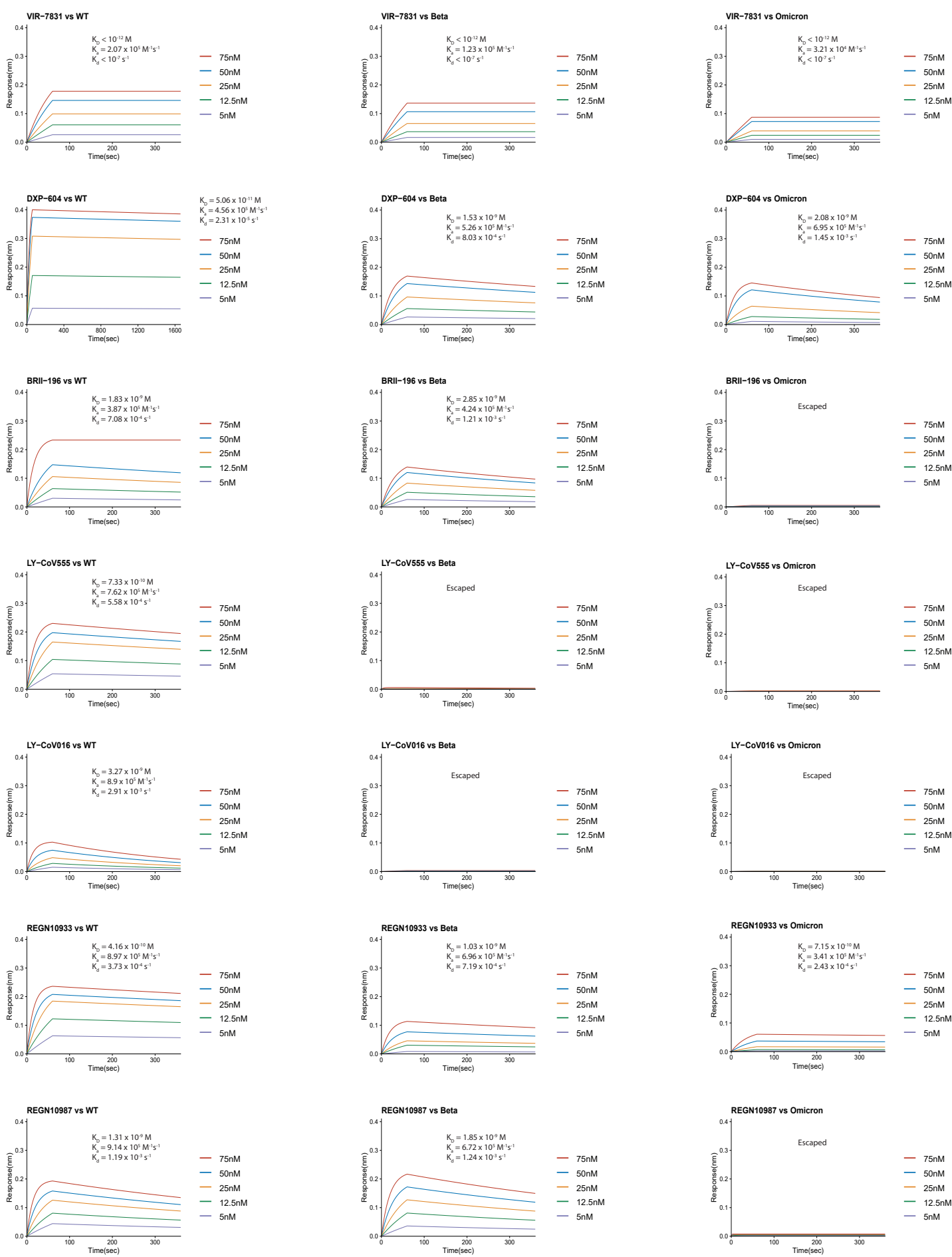

$-\quad 75 \mathrm{n}$
$-\quad 50 \mathrm{M}$
$-\quad 25 \mathrm{M}$
$-\quad 12.5 \mathrm{M}$
$-\quad 5 \mathrm{nM}$
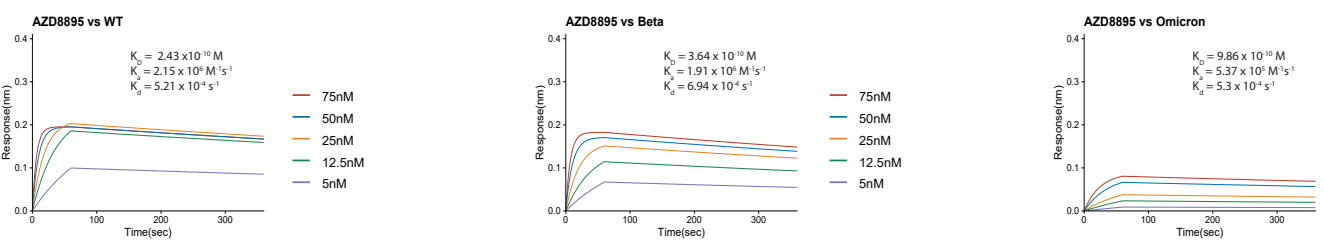

$-\quad 75 \mathrm{nM}$
$-\quad 50 \mathrm{M}$
$-\quad 25 \mathrm{M}$
$-\quad 12.5 \mathrm{n}$
$-\quad 5 \mathrm{mM}$
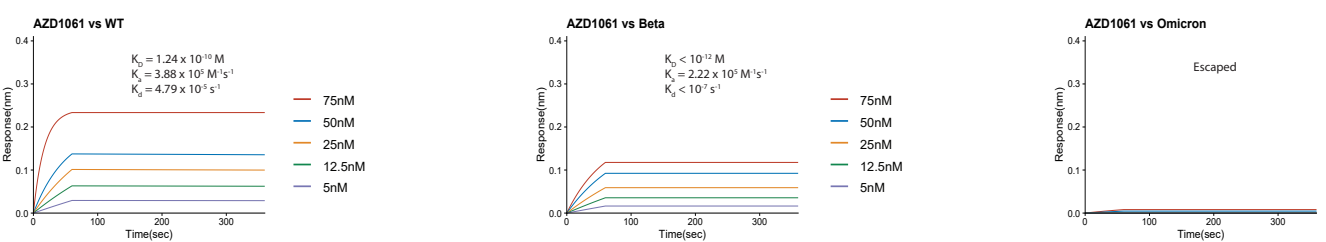

$-75 \mathrm{nM}$

$-\quad 50 \mathrm{MM}$
$-\quad 25 \mathrm{~m}$
$-\quad 50$

$\begin{array}{r}-\quad 25 \mathrm{nM} \\ -\quad 12.5 \mathrm{mM} \\ \hline\end{array}$

$-\quad 12.5 \mathrm{M}$
$-\quad 5 \mathrm{MM}$

Extended Data Fig. 6. Fitted line plot showing the BLI response between antibodies and the receptor binding domain(RBD) of SARS-CoV-2 WT, Beta, or Omicron strain.

The concentrations of RBD are shown in different colors. Dissociation constant(KD), associationconstant $(\mathrm{Ka})$ and dissociation rate constant $(\mathrm{Kd})$ are noted on the figure. The assays without binding are marked as "Escaped". 


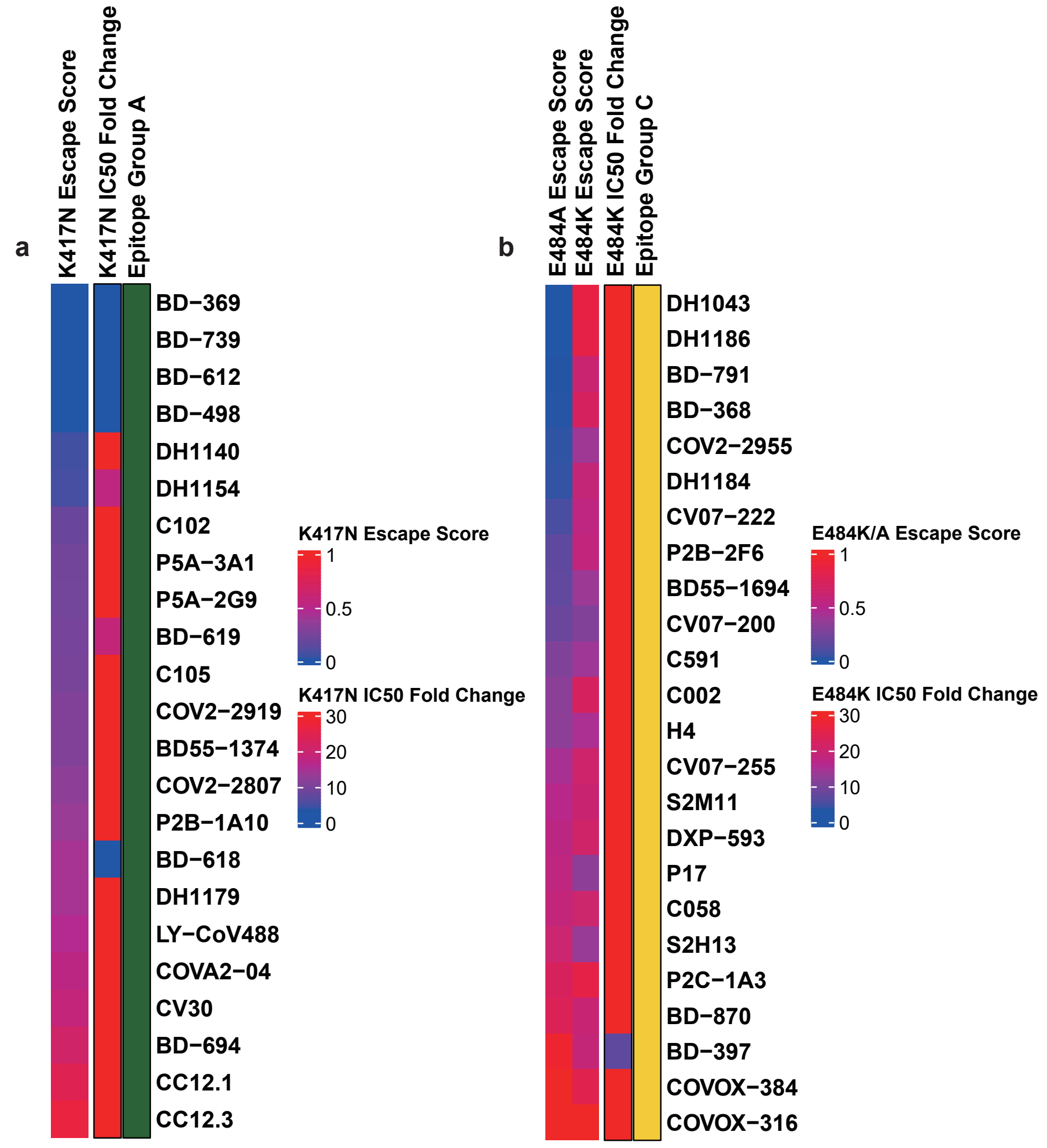

Extended Data Fig. 7 Comparison between mutation escape scores estimated from high throughput deep mutational scanning and neutralization IC50 of pseudovirus with corresponding mutations.

(a) K417N escape scores and corresponding K417N pseudovirus neutralizing IC50 fold change compared to D614G pseudovirus of antibodies within epitope group A.

(b) E484K/E484A escape scores and corresponding E484K pseudovirus neutralizing IC50 fold change compared to $\mathrm{D} 614 \mathrm{G}$ pseudovirus of antibodies within epitope group C. 


\section{Supplementary Files}

This is a list of supplementary files associated with this preprint. Click to download.

- SupplymentaryFile6.xlsx

- Aaalogo.pdf

- Baalogo.pdf

- Caalogo.pdf

- Daalogo.pdf

- Eaalogo.pdf

- Faalogo.pdf 